\title{
The Truth Behind the Myth of the Folk Theorem
}

\author{
Joseph Y. Halpern Rafael Pass Lior Seeman \\ Computer Science Dept. \\ Cornell University \\ Ithaca, NY \\ E-mail: halpern|rafael||seeman@cs.cornell.edu
}

\begin{abstract}
We study the problem of computing an $\epsilon$-Nash equilibrium in repeated games. Earlier work by Borgs et al. 2010 suggests that this problem is intractable. We show that if we make a slight change to their model-modeling the players as polynomial-time Turing machines that maintain state - and make some standard cryptographic hardness assumptions (the existence of public-key encryption), the problem can actually be solved in polynomial time. Our algorithm works not only for games with a finite number of players, but also for constant-degree graphical games.

As Nash equilibrium is a weak solution concept for extensive form games, we additionally define and study an appropriate notion of a subgame-perfect equilibrium for computationally bounded players, and show how to efficiently find such an equilibrium in repeated games (again, making standard cryptographic hardness assumptions).
\end{abstract}

\section{Introduction}

The complexity of finding a Nash equilibrium (NE) is a fundamental question at the interface of game theory and computer science. A celebrated sequence of results showed that the complexity of finding a NE in a normal-form game is PPAD-complete Chen and Deng 2006. Daskalakis, Goldberg, and Papadimitriou 2006, even for 2-player games. Less restrictive concepts, such as $\epsilon$-NE for an inverse-polynomial $\epsilon$, are just as hard Chen, Deng, and Teng 2006. This suggests that these problems are computationally intractable.

There was some hope that the situation would be better in infinitely-repeated games. The Folk Theorem (see Osborne and Rubinstein 1994 for a review) informally states that in an infinitelyrepeated game $G$, for any payoff profile that is individually rational, in that all players get more than 1 their minimax payoff (the highest payoff that a player can guarantee himself, no matter what the other players do) and is the outcome of some correlated strategy in $G$, there is a Nash equilibrium of $G$ with this payoff profile. With such a large set of equilibria, the hope was that finding one would be less difficult. Indeed, Littman and Stone 2005] showed that these ideas can be used to design an algorithm for finding a NE in a two-player repeated game.

Borgs et al. [2010] ( $\mathrm{BC}+$ from now on) proved some results suggesting that, for more than two players, even in infinitely-repeated games it would be difficult to find a NE. Specifically, they showed that, under certain assumptions, the problem of finding a NE (or even an $\epsilon$-NE for an inverse-polynomial $\epsilon$ ) in an infinitely repeated game with three or more players where there is a discount factor bounded away from 1 by an inverse polynomial is also PPAD-hard. They prove this by showing that, given an arbitrary normal-form game $G$ with $c \geq 2$ players, there is a game $G^{\prime}$

\footnotetext{
${ }^{1}$ For our results, since we consider $\epsilon$-NE, we can replace "more than" by "at least".
} 
with $c+1$ players such that finding an $\epsilon / 8 c$-NE for the repeated game based on $G^{\prime}$ is equivalent to finding an $\epsilon$-NE for $G$.

While their proof is indeed correct, in this paper, we challenge their conclusion. If we take seriously the importance of being able to find an $\epsilon$-NE efficiently, it is partly because we have computationally bounded players in mind. But then it seems reasonable to see what happens if we assume that the players in the game are themselves computationally bounded. Like $\mathrm{BC}+$, we assume that players are resource bounded $\sqrt{2}$ Formally, we view players as probabilistid 3 polynomial-time Turing machines (PPT TMs). We differ from BC+ in two key respects. First, since we restrict to (probabilistic) polynomial-time players, we restrict the deviations that can be made in equilibrium to those that can be computed by such players; BC+ allow arbitrary deviations. Second, BC+ implicitly assume that players have no memory: they cannot remember computation from earlier rounds. By way of contrast, we allow players to have a bounded (polynomial) amount of memory. This allows players to remember the results of a few coin tosses from earlier rounds, and means that we can use some cryptography (making some standard cryptographic assumptions) to try to coordinate the players. We stress that this coordination happens in the process of the game play, not through communication. That is, there are no side channels; the only form of "communication" is by making moves in the game. We call such TMs stateful, and the BC+ TMs stateless. We note, that without the restriction on deviations, there is no real difference between stateful TMs and stateless TMs in our setting (since a player with unbounded computational power can recreate the necessary state). With these assumptions (and the remaining assumptions of the BC+ model), we show that in fact an $\epsilon$-NE in an infinitely-repeated game can be found in polynomial time.

Our equilibrium strategy uses threats and punishment much in the same way that they are used in the Folk Theorem. However, since the players are computationally bounded we can use cryptography (we assume the existence of a secure public key encryption scheme) to secretly correlate the punishing players. This allows us to overcome the difficulties raised by $\mathrm{BC}+$. Roughly speaking, the $\epsilon$-NE can be described as proceeding in three stages. In the first stage, the players play a sequence of predefined actions repeatedly. If some player deviates from the sequence, the second stage begins, in which the other players use their actions to secretly exchange a random seed, through the use of public-key encryption. In the third stage, the players use a correlated minimax strategy to punish the deviator forever. To achieve this correlation, the players use the secret random seed as the seed of a pseudorandom function, and use the outputs of the pseudorandom function as the source of randomness for the correlated strategy. Since the existence of public-key encryption implies the existence of pseudorandom functions, the only cryptographic assumption needed is the existence of public-key encryptions - one of the most basic cryptographic hardness assumptions.

In the second part of the paper we show how to extend this result to a more refined solution concept. While NE has some attractive features, it allows some unreasonable solutions. In particular, the equilibrium might be obtained by what are arguably empty threats. This actually happens in our proposed NE (and in the basic version of the folk theorem). Specifically, players are required to punish a deviating player, even though that might hurt their payoff. Thus, if a deviation occurs, it might not be the best response of the players to follow their strategy and punish; thus, such a punishment is actually an empty threat.

To deal with this (well known) problem, a number of refinements of NE have been considered. The one typically used in dynamic games of perfect information is subgame-perfect equilibrium, suggested by Selten [1965]. A strategy profile is a subgame-perfect equilibrium if it is a NE at

\footnotetext{
${ }^{2}$ Although $\mathrm{BC}+$ do not discuss modeling players in this way, the problem they show is NP-Hard is to find a polynomial-time TM profile that implements an equilibrium. There is an obvious exponential-time TM profile that implements an equilibrium: each TM in the profile just computes the single-shot NE and plays its part repeatedly.

${ }^{3} \mathrm{BC}+$ describe their TMs as deterministic, but allow them to output a mixed strategy. As they point out, there is no difference between this formulation and a probabilistic TM that outputs a specific action; their results hold for such probabilistic TMs as well.
} 
every subgame of the original game. Informally, this means that at any history of the game (even those that are not on any equilibrium path), if all the players follow their strategy from that point on, then no player has an incentive to deviate. In the context of repeated games where players' moves are observed (so that it is a game of perfect information), the folk theorem continues to hold even if the solution concept used is subgame-perfect equilibrium Aumann and Shapley 1994; Fudenberg and Maskin 1986; Rubinstein 1979.

We define a computational analogue of subgame-perfect equilibrium that we call computational subgame-perfect $\epsilon$-equilibrium, where the strategies involved are polynomial time, and deviating players are again restricted to using polynomial-time strategies. There are a number of subtleties that arise in defining this notion. While we assume that all actions in the underlying repeated game are observable, we allow our TMs to also have memory, which means the action of A TM does not depend only on the public history. Like subgame-perfect equilibrium, our computational solution concept is intended to capture the intuition that the strategies are in equilibrium after any possible deviation. This means that in a computational subgame-perfect equilibrium, at each history for player $i$, player $i$ must make a (possibly approximate) best response, no matter what his and the other players' memory states are.

To compute a computational subgame-perfect $\epsilon$-equilibrium, we use the same basic strategy as for NE, but, as often done to get a subgame-perfect equilibrium (for example see Fudenberg and Maskin 1986), we limit the punishment phase length, so that the players are not incentivized not to punish deviations. However, to prove our result, we need to overcome one more significant hurdle. When using cryptographic protocols, it is often the case (and, specifically is the case in the protocol used for NE) that player $i$ chooses a secret (e.g., a secret key for a public-key encryption scheme) as the result of some randomization, and then releases some public information which is a function of the secret (e.g., a public key). After that public information has been released, another party $j$ typically has a profitable deviation by switching to the TM $M$ that can break the protocol - for every valid public information, there always exists some TM $M$ that has the secret "hardwired" into it (although there may not be an efficient way of finding $M$ given the information). We deal with this problem by doing what is often done in practice: we do not use any key for too long, so that $j$ cannot gain too much by knowing any one key.

A second challenge we face is that in order to prove that our new proposed strategies are even an $\epsilon$-NE, we need to show that the payoff of the best response to this strategy is not much greater than that of playing the strategy. However, since for any polynomial-time TM there is always a better polynomial-time TM that has just a slightly longer running time, this natural approach fails. This instead leads us to characterize a class of TMs we can analyze, and show that any other TM can be converted to a TM in this class that has at least the same payoff. While such an argument might seem simple in the traditional setting, since we only allow for polynomial time TMs, in our setting this turns out to require a surprisingly delicate construction and analysis to make sure this converted TM does indeed has the correct size and running time.

The idea of considering resource-bounded agents has a long history in game theory. It is known, for example, that cooperation is a NE of finitely-repeated prisoner's dilemma with resource-bounded players (see, e.g., [Neyman 1985; Rubinstein 1986; Papadimitriou and Yannakakis 1994]). The idea of using the structure of the game as a means of correlation is used by Lehrer [1991 to show an equivalence between $\mathrm{NE}$ and correlated equilbrium in certain repeated games with nonstandard information structures. The use of cryptography in game theory goes back to Urbano and Vila 2002, 2004, who also used it to do coordination between players. More recently, it has been used by, for example, Dodis, Halevi, and Rabin [2000].

The application of cryptography perhaps most closely related to ours is by Gossner [1998], who uses cryptographic techniques to show how any payoff profile that is above the players' correlated minimax value can be achieved in a $\mathrm{NE}$ of a repeated game with public communication played by 
computationally bounded players. In Gossner 2000], a strategy similar to the one that we use is used to prove that, even without communication, the same result holds. Gossner's results apply only to infinitely-repeated games with 3 players and no discounting; he claims that his results do not hold for games with discounting. Gossner does not discuss the complexity of finding a strategy of the type that he shows exists.

Recently, Andersen and Conitzer 2013 described an algorithm for finding NE in repeated games with more than two players with high probability in uniform games. However, this algorithm is not guaranteed to work for all games, and uses the limit of means as its payoff criterion, and not discounting.

There are a few recent papers that investigate solution concepts for extensive-form games involving computationally bounded player Kol and Naor 2008; Gradwohl, Livne, and Rosen 2013, Halpern and Pass 2013]; some of these focus on cryptographic protocols [Kol and Naor 2008, Gradwohl, Livne, and Rosen 2013. Kol and Naor 2008 discuss refinements of NE in the context of cryptographic protocols, but their solution concept requires only that on each history on the equilibrium path, the strategies from that point on form a NE. Our requirement for the computational subgame-perfect equilibrium is much stronger. Gradwohl, Livne and Rosen [2013] also consider this scenario and offer a solution concept different from ours; they try to define when an empty threat occurs, and look for strategy profiles where no empty threats are made. Again, our solution concept is much stronger.

The rest of this paper is organized as follows. In Section 2, we review the relevant definitions from game theory and cryptography. In section 3, we define our notion of computational $\epsilon$-NE and show how find it efficiently for repeated games. In Section 4, we consider computational subgame-perfect $\epsilon$-equilibrium and show that it too can be found efficiently.

\section{Preliminaries}

\subsection{One-shot games}

We define a game $G$ to be a triple $([c], A, \vec{u})$, where $[c]=\{1, \ldots, c\}$ is the set of players, $A_{i}$ is the set of possible actions for player $i, A=A_{1} \times \ldots \times A_{c}$ is the set of action profiles, and $\vec{u}: A \rightarrow \mathbb{R}^{c}$ is the utility function $\left(\vec{u}_{i}(\vec{a})\right.$ is the utility of player $i$ ). A (mixed) strategy $\sigma_{i}$ for player $i$ is a probability distribution over $A_{i}$, that is, an element of $\Delta\left(A_{i}\right)$ (where, as usual, we denote by $\Delta(X)$ the set of probability distributions over the set $X)$. We use the standard notation $\vec{x}_{-i}$ to denote vector $\vec{x}$ with its $i$ th element removed, and $\left(x^{\prime}, \vec{x}_{-i}\right)$ to denote $\vec{x}$ with its $i$ th element replaced by $x^{\prime}$.

Definition 2.1. (Nash Equilibrium) $\sigma=\left(\sigma_{1}, \ldots, \sigma_{c}\right)$ is an $\epsilon-N E$ of $G$ if, for all players $i \in[c]$ and all actions $a_{i}^{\prime} \in A_{i}, E_{\sigma_{-i}}\left[u_{i}\left(a_{i}^{\prime}, \vec{a}_{-i}\right)\right] \leq E_{\sigma}\left[u_{i}(\vec{a})\right]+\epsilon$.

A correlated strategy of a game $G$ is an element $\sigma \in \Delta(A)$. It is a correlated equilibrium if, for all players $i$, they have no temptation to play a different action, given that the action profile was chosen according to $\sigma$. That is, for all players $i$ for all $a_{i} \in A_{i}$ such that $\sigma_{i}\left(a_{i}\right)>0, E_{\sigma \mid a_{i}} u_{i}\left(a_{i}, \vec{a}_{-i}\right) \geq$ $E_{\sigma \mid a_{i}} u_{i}\left(a_{i}^{\prime}, \vec{a}_{-i}\right)$.

Player $i$ 's minimax value in a game $G$ is the highest payoff $i$ can guarantee himself if the other players are trying to push his payoff as low as possible. We call the strategy $i$ plays in this case a minimax strategy for $i$; the strategy that the other players use is $i$ 's (correlated) punishment strategy. (Of course, there could be more than one minimax strategy or punishment strategy for player $i$.) Note that a correlated punishment strategy can be computed using linear programming.

Definition 2.2. Given a game $G=([c], A, \vec{u})$, the strategies $\vec{\sigma}_{-i} \in \Delta\left(A_{-i}\right)$ that minimize $\max _{\sigma^{\prime} \in \Delta\left(A_{i}\right)} E_{\left(\sigma^{\prime}, \vec{\sigma}_{-i}\right)}\left[u_{i}(\vec{a})\right]$ are the punishment strategies against player $i$ in $G$. If $\vec{\sigma}_{-i}$ is a punishment strategy against player $i$, then $m_{i}(G)=\max _{a \in A_{i}} E_{\vec{\sigma}_{-i}}\left[u_{i}\left(a, a_{-i}\right)\right]$ is player $i$ 's minimax value in $G$ 
To simplify the presentation, we assume all payoffs are normalized so that each player's minimax value is 0 . Since, in an equilibrium, all players get at least their minimax value, this guarantees that all players get at least 0 in a NE.

\subsection{Infinitely repeated games}

Given a normal-form game $G$, we define the repeated game $G^{t}(\delta)$ as the game in which $G$ is played repeatedly $t$ times (in this context, $G$ is called the stage game) and $1-\delta$ is the discount factor (see below). Let $G^{\infty}(\delta)$ be the game where $G$ is played infinitely many times. An infinite history $h$ in this game is an infinite sequence $\left\langle\vec{a}^{0}, \vec{a}^{1}, \ldots\right\rangle$ of action profiles. Intuitively, we can think of $\vec{a}^{t}$ as the action profile played in the $t^{\text {th }}$ stage game. We often omit the $\delta$ in $G^{\infty}(\delta)$ if it is not relevant to the discussion. Let $H_{G^{\infty}}$ be the set of all possible histories of $G^{\infty}$. For a history $h \in H_{G^{\infty}}$ let $G^{\infty}(h)$ the subgame that starts at history $h$ (after $|h|$ one-shot games have been played where all players played according to $h$ ). We assume that $G^{\infty}$ is fully observable, in the sense that, after each stage game, the players observe exactly what actions the other players played.

A (behavioral) strategy for player $i$ in a repeated game is a function $\sigma$ from histories of the games to $\Delta\left(A_{i}\right)$. Note that a profile $\vec{\sigma}$ induces a distribution $\rho_{\vec{\sigma}}$ on infinite histories of play. Let $\rho_{\vec{\sigma}}^{t}$ denote the induced distribution on $H^{t}$, the set of histories of length $t$. (If $t=0$, we take $H^{0}$ to consist of the unique history of length 0 , namely \langle\rangle .) Player $i$ 's utility if $\vec{\sigma}$ is played, denoted $p_{i}(\vec{\sigma})$, is defined as follows:

$$
p_{i}(\vec{\sigma})=\delta \sum_{t=0}^{\infty}(1-\delta)^{t} \sum_{h \in H^{t}, \vec{a} \in A} \rho_{\vec{\sigma}}^{t+1}(h \cdot \vec{a})\left[u_{i}(\vec{a})\right] .
$$

Thus, the discount factor is $1-\delta$. Note that the initial $\delta$ is a normalization factor. It guarantees that if $u_{i}(\vec{a}) \in\left[b_{1}, b_{2}\right]$ for all joint actions $\vec{a}$ in $G$, then $i$ 's utility is in $\left[b_{1}, b_{2}\right]$, no matter which strategy profile $\vec{\sigma}$ is played.

In these game, a more robust solution concept is subgame-perfect equilibrium [Selten 1965], which requires that the strategies form an $\epsilon$-NE at every history of the game.

Definition 2.3. A strategy profile $\vec{\sigma}=\left(\sigma_{1}, \ldots, \sigma_{c}\right)$, is a subgame-perfect $\epsilon$-equilibrium of a repeated game $G^{\infty}$, if, for all players $i \in[c]$, all histories $h \in H_{G^{\infty}}$ where player $i$ moves, and all strategies $\sigma^{\prime}$ for player $i$,

$$
p_{i}^{h}\left(\left(\sigma^{\prime}\right)^{h}, \vec{\sigma}_{-i}^{h}\right) \leq p_{i}^{h}\left(\vec{\sigma}^{h}\right)+\epsilon,
$$

where $p_{i}^{h}$ is the utility function for player $i$ in game $G^{\infty}(h)$, and $\sigma^{h}$ is the restriction of $\sigma$ to $G^{\infty}(h)$.

\subsection{Cryptographic definitions}

For a probabilistic algorithm $A$ and an infinite bit string $r, A(x ; r)$ denotes the output of $A$ running on input $x$ with randomness $r ; A(x)$ denotes the distribution on outputs of $A$ induced by considering $A(x ; r)$, where $r$ is chosen uniformly at random. A function $\epsilon: \mathbb{N} \rightarrow[0,1]$ is negligible if, for every constant $c \in \mathbb{N}, \epsilon(k)<k^{-c}$ for sufficiently large $k$.

We use a non-uniform security model, which means our attackers are non-uniform PPT algorithm.

Definition 2.4. A non-uniform probabilistic polynomial-time machine $A$ is a sequence of probabilistic machines $A=\left\{A_{1}, A_{2}, \ldots\right\}$ for which there exists a polynomial d such that both $\left|A_{n}\right|$, the description size of $A_{n}$ (i.e., the states and transitions in $A_{n}$ ), and the running time of $A_{n}$ are less than $d(i)$.

Alternatively, a non-uniform PPT machine can also be defined as a uniform PPT machine that receives an advice string (for example, on an extra "advice" tape) for each input length. It is common 
to assume that the cryptographic building blocks we define next and use in our constructions are secure against non-uniform PPT algorithms.

\subsubsection{Computational Indistinguishability}

Definition 2.5. A probability ensemble is a sequence $X=\left\{X_{n}\right\}_{n \in \mathbb{N}}$ of probability distribution indexed by $\mathbb{N}$. (Typically, in an ensemble $X=\left\{X_{n}\right\}_{n \in \mathbb{N}}$, the support of $X_{n}$ consists of strings of length n.)

We now recall the definition of computational indistinguishability [Goldwasser and Micali 1984].

Definition 2.6. Two probability ensembles $\left\{X_{n}\right\}_{n \in \mathbb{N}},\left\{Y_{n}\right\}_{n \in \mathbb{N}}$ are computationally indistinguishable if, for all non-uniform PPT TMs D, there exists a negligible function $\epsilon$ such that, for all $n \in \mathbb{N}$,

$$
\left|\operatorname{Pr}\left[D\left(1^{n}, X_{n}\right)=1\right]-\operatorname{Pr}\left[D\left(1^{n}, Y_{n}\right)=1\right]\right| \leq \epsilon(n) .
$$

To explain the $\operatorname{Pr}$ in the last line, recall that $X_{n}$ and $Y_{n}$ are probability distributions. Although we write $D\left(1^{n}, X_{n}\right), D$ is a randomized algorithm, so what $D\left(1^{n}, X_{n}\right)$ returns depends on the outcome of random coin tosses. To be a little more formal, we should write $D\left(1^{n}, X_{n}, r\right)$, where $r$ is an infinitely long random bit strong (of which $D$ will only use a finite initial prefix). More formally, taking $\operatorname{Pr}_{X_{n}}$ to be the joint distribution over strings $(x, r)$ where $x$ is chosen according to $X_{n}$ and $r$ is chosen according to the uniform distribution on bit-strings, we want

$$
\left|\operatorname{Pr}_{X_{n}}\left[\left\{(x, r): D\left(1^{n}, x, r\right)=1\right\}\right]-\operatorname{Pr}_{Y_{n}}\left[\left\{(y, r): D\left(1^{n}, y, r\right)=1\right\}\right]\right| \leq \epsilon(n) .
$$

We similarly abuse notation elsewhere in writing $\mathrm{Pr}$.

We often call a TM that is supposed to distinguish between two probability ensembles a distinguisher.

\subsubsection{Pseudorandom Functions}

Definition 2.7. A function ensemble is a sequence $F=\left\{F_{n}\right\}_{n \in \mathbb{N}}$ of probability distributions such that the support of $F_{n}$ is a set of functions mapping n-bit strings to $n$-bit strings. The uniform function ensemble, denoted $H=\left\{H_{n}\right\}_{n \in \mathbb{N}}$, has $H_{n}$ be the uniformly distribution over the set of all functions mapping $n$-bit strings to $n$-bit strings.

We have the same notion of computational indistinguishablity for function ensembles as we had for probability ensembles, only that the distinguisher is now an oracle machine, meaning that it can query the value of the function at any point with one computation step, although it does not have the full description of the function. (See Goldreich 2001 for a detailed description.)

We now define pseudorandom functions (see Goldreich, Goldwasser, and Micali 1986). Intuitively, this is a family of functions indexed by a seed, such that it is hard to distinguish a random member of the family from a truly randomly selected function.

Definition 2.8. A pseudorandom function ensemble (PRF) is a set $\left\{f_{s}:\{0,1\}^{|s|} \rightarrow\{0,1\}^{|s|}\right\}_{s \in\{0,1\}^{*}}$ such that the following conditions hold:

- (easy to compute) $f_{s}(x)$ can be computed by a PPT algorithm that is given s and $x$;

- (pseudorandom) the function ensemble $F=\left\{F_{n}\right\}_{n \in \mathbb{N}}$, where $F_{n}$ is uniformly distributed over the multiset $\left\{f_{s}\right\}_{s \in\{0,1\}^{n}}$, is computationally indistinguishable from $H$. 
We use the standard cryptographic assumption that a family of PRFs exists; this assumption is implied by the existence of one-way functions Håstad, Impagliazzo, Levin, and Luby 1999; Goldreich, Goldwasser, and Micali 1986. We actually require the use of a seemingly stronger notion of a PRF, which requires that an attacker getting access to polynomially many instances of a PRF (i.e., $f_{s}$ for polynomially many values of $s$ ) still cannot distinguish them from polynomially many truly random functions. Nevertheless, as we show in Appendix $\mathrm{A}$, it follows using a standard "hybrid" argument that any PRF satisfies also this stronger "multi-instance" security notion.

\subsubsection{Public-key Encryption Schemes}

We now define public-key encryption schemes. Such a scheme has two keys. The first is public and used for encrypting messages (using a randomized algorithm). The second is secret and used for decrypting. The keys are generated in such a way that the probability that a decrypted message is equal to the encrypted message is equal to 1 . The key generation algorithm takes as input a "security parameter" $k$ that is used to determine the security of the protocols (inuitively, no polynomial-time attacker should be able to "break" the security of the protocol except possibly with a probability that is a negligible function of $k$ ).

We now recall the formal definitions of public-key encryption schemes [Diffie and Hellman 1976, Rivest, Shamir, and Adleman 1978; Goldwasser and Micali 1984].

Definition 2.9. Given a polynomial $l$, an l-bit public-key encryption scheme is a triple $\Pi=\left(\right.$ Gen,Enc,Dec) of PPT algorithms where (a) Gen takes a security parameter $1^{k}$ as input and returns a (public key, private key) pair; (b) Enc takes a public key pk and a message $m$ in a message space $\{0,1\}^{l(k)}$ as input and returns a ciphertext $\operatorname{Enc}_{p k}(m)$; (c) Dec is a deterministic algorithm that takes a secret key sk and a ciphertext $\mathcal{C}$ as input and outputs $m^{\prime}=D e c_{s k}(\mathcal{C})$, and (d)

$$
\operatorname{Pr}\left[\exists m \in\{0,1\}^{l(k)} \text { such that } \operatorname{Dec}_{s k}\left(\operatorname{Enc}_{p k}(m)\right) \neq m\right]=0 .
$$

We next define a security notion for public-key encryption. Such a security notion considers an adversary that is characterized by two PPT algorithms, $A_{1}$ and $A_{2}$. Intuitively, $A_{1}$ gets as input a public key that is part of a (public key, secret key) pair randomly generated by Gen, together with a security parameter $k$. $A_{1}$ then outputs two messages in $\{0,1\}^{k}$ (intuitively, messages it can distinguish), and some side information that it passes to $A_{2}$ (intuitively, this is information that $A_{2}$ needs, such as the messages chosen; An example of how this is used can be seen in Appendix (B)). $A_{2}$ gets as input the encryption of one of those messages and the side information passed on by $A_{1}$. $A_{2}$ must output which of the two messages $m_{0}$ and $m_{1}$ the encrypted message is the encryption of (where an output of $b \in\{0,1\}$ indicates that it is $m_{b}$ ). Since $A_{1}$ and $A_{2}$ are PPT algorithms, the output of $A_{2}$ can be viewed as a probability distribution over $\{0,1\}$. The scheme is secure if the two ensembles (i.e., the one generated by this process where the encryption of $m_{0}$ is always given to $A_{2}$, and the one where the encryption of $m_{1}$ is always given to $A_{2}$ ) are indistinguishable. More formally:

Definition 2.10 (Public-key security). An l-bit public-key encryption scheme $\Pi=($ Gen, Enc, Dec) is secure if, for every probabilistic polynomial-time adversary $A=\left(A_{1}, A_{2}\right)$, the ensembles $\left\{\operatorname{IND}_{0}^{\Pi}(A, k)\right\}_{k}$ and $\left\{\operatorname{IND}_{1}^{\Pi}(A, k)\right\}_{k}$ are computationally indistinguishable, where $\left\{\operatorname{IND}_{b}^{\Pi}(A, k)\right\}_{k}$ is the following PPT algorithm:

$$
\begin{aligned}
I N D_{b}^{\Pi}(A, k):= & (p k, s k) \leftarrow \operatorname{Gen}\left(1^{k}\right) \\
& \left(m_{0}, m_{1}, \tau\right) \leftarrow A_{1}\left(1^{k}, p k\right)\left(m_{0}, m_{1} \in\{0,1\}^{k}\right) \\
& \mathcal{C} \leftarrow \text { Enc } c_{p k}\left(m_{b}\right) \\
& \text { o } \leftarrow A_{2}(\mathcal{C}, \tau) \\
& \text { Output } 0 .
\end{aligned}
$$


Intuitively, the $\leftarrow$ above functions as an assignment statement, but it is not quite that, since the various algorithms are actually PPT algorithms, so their output is randomized. Formally, $I N D_{b}^{\Pi}(A, k)$ is a probability distribution, which we can write as $\operatorname{IND}_{b}^{\Pi}\left(A, k, r_{1}, r_{2}, r_{3}, r_{4}\right)$, where we view $r_{1}, r_{2}$, $r_{3}$, and $r_{4}$ as the random bitstrings that serve as the second arguments of Gen, $A_{1}$, Enc $c_{p k}$, and $A_{2}$, respectively. Once we add these arguments (considering, e.g., Gen $\left(1^{k}, r_{1}\right)$ and $A_{1}\left(1^{k}, p k, r_{2}\right)$ rather than $G e n\left(1^{k}\right)$ and $\left.A_{1}\left(1^{k}, p k\right)\right)$ these algorithms become deterministic, and $\leftarrow$ can indeed be viewed as an assignment statement.

We assume a secure public-key encryption scheme exists. We actually require a seemingly stronger notion of "multi-instance" security, where an attacker gets to see encryptions of multiple messages, each of which is encrypted using multiple keys.

Definition 2.11. An l-bit public-key encryption scheme $\Pi=($ Gen,Enc,Dec $)$ is multi-message multi-key secure if, for all polynomials $f$ and $g$, and for every probabilistic polynomial time adver-

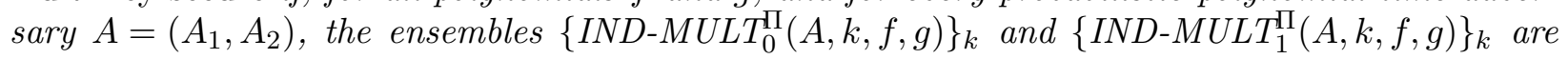
computationally indistinguishable, where

$$
\begin{aligned}
& I N D-M U L T_{b}^{\Pi}(A, k, f, g):= \\
& \left(p k_{1}, s k_{1}\right) \leftarrow \operatorname{Gen}\left(1^{k}\right), \ldots\left(p k_{g(k)}, s k_{g(k)}\right) \leftarrow \operatorname{Gen}\left(1^{k}\right), \\
& \left(m_{0}^{1}, \ldots, m_{0}^{f(k)}, m_{1}^{1}, \ldots, m_{1}^{f(k)}, \tau\right) \leftarrow A_{1}\left(1^{k}, p k_{1}, \ldots, p k_{g(k)}\right)\left(m_{0}^{i}, m_{1}^{i} \in\{0,1\}^{k}\right) \\
& \mathcal{C} \leftarrow E n c_{p k_{1}}\left(m_{b}^{1}\right), \ldots, E n c_{p k_{g(k)}}\left(m_{b}^{1}\right), \ldots, E n c_{p k_{1}}\left(m_{b}^{f(k)}\right), \ldots, E n c_{p k_{g(k)}}\left(m_{b}^{f(k)}\right) \\
& o \leftarrow A_{2}(\mathcal{C}, \tau) \\
& \text { Output } o
\end{aligned}
$$

In this definition, there are polynomially many messages being encrypted, and each message is encrypted a polynomial number of times, using a different key each time. Other than that, the process is similar to the standard definition of security. As we show in Appendix B, any secure encryption scheme is also multi-message multi-key secure.

\section{The complexity of finding $\epsilon-\mathrm{NE}$ in repeated games played by stateful machines}

\subsection{Computational NE Definition}

Since we consider computationally-bounded players, we take a player's strategy in $G^{\infty}$ to be a (possibly probabilistic) Turing machine (TM), which outputs at each round an action to be played, based on its internal memory and the history of play so far. (The TMs considered in BC+ did not have internal memory.) We consider only TMs that at round $t$ use polynomial in $n t$ many steps to compute the next action, where $n$ is the maximum number of actions a player has in $G$. Thus, $n$ is a measure of the size of $G$ 出 Denote by $M_{i}$ the TM used by player $i$, and let $\vec{M}=\left(M_{1}, \ldots, M_{c}\right)$.

Note that a profile $\vec{M}$ induces a distribution $\rho_{\vec{M}}$ on infinite histories of play. Let $\rho_{\vec{M}}^{t}$ denote the induced distribution on $H^{t}$, the set of histories of length $t$. (If $t=0$, we take $H^{0}$ to consist of the unique history of length 0 , namely \langle\rangle .) Player $i$ 's utility if $\vec{M}$ is played, denoted $p_{i}(\vec{M})$, is defined as follows:

$$
p_{i}(\vec{M})=\delta \sum_{t=0}^{\infty}(1-\delta)^{t} \sum_{h \in H^{t}, \vec{a} \in A} \rho_{\vec{M}}^{t+1}(h \cdot \vec{a})\left[u_{i}(\vec{a})\right] .
$$

\footnotetext{
${ }^{4}$ When we talk about polynomial-time algorithms, we mean polynomial in $n$. We could use other measures of the size of $G$, such as the total number of actions. Since all reasonable choices of size are polynomially related, the choice does not affect our results.
} 
Thus, the discount factor is $1-\delta$. Note that the initial $\delta$ is a normalization factor. It guarantees that if $u_{i}(\vec{a}) \in\left[b_{1}, b_{2}\right]$ for all joint actions $\vec{a}$ in $G$, then $i$ 's utility is in $\left[b_{1}, b_{2}\right]$, no matter which TM profile $\vec{M}$ is played.

We are now ready to define the notion of equilibrium we use. Intuitively, as we model players as polynomial-time TMs, we consider a profile of TMs an equilibrium in a game if there is no player and no other polynomial-time TM that gives that player a higher expected payoff (or up to an $\epsilon$ for an $\epsilon$-NE).

Since we consider (probabilistic) TMs that run in polynomial time in the size of the game, we cannot consider a single game. For any fixed game, running in polynomial time in the size of the game is meaningless. Instead, we need to consider a sequence of games. This leads to the following definition.

Definition 3.1. An infinite sequence of strategy profiles $\vec{M}^{1}, \vec{M}^{2}, \ldots$, where $\vec{M}^{k}=\left(M_{1}^{k}, \ldots, M_{c}^{k}\right)$ is an $\epsilon-N E$ of an infinite sequence of games $G_{1}^{\infty}, G_{2}^{\infty}, \ldots$ where the size of $G_{k}$ is $k$ if, for all players $i \in[c]$ and all non-uniform PPT adversaries $\bar{M}$ (polynomial in $k$ and $t$, as discussed above), there exist $k_{0}$ such that for all $k \geq k_{0}$

$$
p_{i}^{k}\left(\bar{M}, \vec{M}_{-i}^{k}\right) \leq p_{i}^{k}\left(\vec{M}^{k}\right)+\epsilon(k) .
$$

where $p_{i}^{k}$ is the payoff of player $i$ in game $G_{k}^{\infty}$.

We note that the equilibrium definition we use considers only deviations that can be implemented by non-uniform polynomial-time TMs. This is different from both the usual definition of $\mathrm{NE}$ and from the definition used by $\mathrm{BC}+$, who allow arbitrary deviations. But this difference is exactly what allows us to use cryptographic techniques. The need to define polynomial-time deviation is the reason for considering sequences of games instead of a single game. There are other reasonable ways of capturing polynomial-time adversaries. As will be seen from our proof, our approach is quite robust, so our results should hold for any reasonable definition.

\subsection{Computing an equilibrium}

In this section we describe the equilibrium strategy and show how to efficiently compute it. We first start with some definition and tool we need for our proof.

\subsubsection{Preliminaries}

Definition 3.2. Let $\mathcal{G}_{a, b, c, n}$ be the set of all games with c players, at most $n$ actions per player, integral payoff 5 , maximum payoff $a$, and minimum payoff $b$.

Note that by our assumption that the minimax payoff is 0 for all players, we can assume $a \geq 0$, $b \leq 0$, and $a-b>0$ (otherwise $a=b=0$, which makes the game uninteresting). We start by showing that, given a correlated strategy $\sigma$ in a game $G$, players can get an average payoff that is arbitrarily close to their payoff in $\sigma$ by playing a fixed sequence of action profiles repeatedly.

Lemma 3.3. For all $a, b, c$, all polynomials $q$, all $n$, all games $G \in \mathcal{G}_{a, b, c, n}$, and all correlated strategies $\sigma$ in $G$, if the expected payoff vector of playing $\sigma$ is $p$ then there exists a sequence sq of length $w(n)$, where $w(n)=((a-b) q(n)+1) n^{c}$, such that player $i$ 's average payoff in $s q$ is at least $p_{i}-1 / q(n)$.

\footnotetext{
${ }^{5}$ Our result also hold for rational payoffs except then the size of the game needs to take into account the bits needed to represent the payoffs
} 
Proof. Given $\sigma$, we create $s q$ the obvious way: by playing each action in proportion to the probability $\sigma(\vec{a})$. More precisely, let $r=a-b$, and define $w(n)=(r q(n)+1) n^{c}$, as in the statement of the lemma. We create a sequence $s q$ by playing each action profile $\vec{a}\lfloor w(n) \sigma(\vec{a})\rfloor$ times, in some fixed order. Notice that the length of this sequence is between $w(n)-n^{c}$ and $w(n)$. The average payoff player $i$ gets in $s q$ is

$$
\begin{aligned}
v_{i}^{\prime} & =\frac{1}{\sum_{\vec{a} \in A}\lfloor w(n) \sigma(\vec{a})\rfloor} \sum_{\vec{a} \in A}\lfloor w(n) \sigma(\vec{a})\rfloor u_{i}(\vec{a}) \\
& \geq \frac{1}{\sum_{\vec{a} \in A}\lfloor w(n) \sigma(\vec{a})\rfloor}\left(\sum_{\vec{a} \in A, u_{i}(\vec{a}) \geq 0}(w(n) \sigma(\vec{a})-1) u_{i}(\vec{a})+\sum_{\vec{a} \in A, u_{i}(\vec{a})<0} w(n) \sigma(\vec{a}) u_{i}(\vec{a})\right) \\
& =\frac{w(n) \sum_{\vec{a} \in A} \sigma(\vec{a}) u_{i}(\vec{a})}{\sum_{\vec{a} \in A}\lfloor w(n) \sigma(\vec{a})\rfloor}-\frac{\sum_{\vec{a} \in A, u_{i}(\vec{a}) \geq 0} u_{i}(\vec{a})}{\sum_{\vec{a} \in A}\lfloor w(n) \sigma(\vec{a})\rfloor} \geq \frac{w(n) p_{i}}{\sum_{\vec{a} \in A}\lfloor w(n) \sigma(\vec{a})\rfloor}-\frac{a n^{c}}{w(n)-n^{c}} .
\end{aligned}
$$

If $p_{i}<0$,

$$
\begin{aligned}
v_{i}^{\prime} & \geq \frac{w(n) p_{i}}{\sum_{\vec{a} \in A}\lfloor w(n) \sigma(\vec{a})\rfloor}-\frac{a n^{c}}{w(n)-n^{c}} \geq \frac{w(n) p_{i}-a n^{c}}{w(n)-n^{c}} \\
& =\frac{(r q(n)+1) n^{c} p_{i}-a n^{c}}{(r q(n)+1) n^{c}-n^{c}}=\frac{r q(n) n^{c} p_{i}-\left(a-p_{i}\right) n^{c}}{r q(n) n^{c}} \geq p_{i}-\frac{1}{q(n)} .
\end{aligned}
$$

If $p_{i} \geq 0$,

$$
\begin{aligned}
v_{i}^{\prime} & \geq \frac{w(n) p_{i}}{\sum_{\vec{a} \in A}\lfloor w(n) \sigma(\vec{a})\rfloor}-\frac{a n^{c}}{w(n)-n^{c}} \geq p_{i}-\frac{a n^{c}}{w(n)-n^{c}} \\
& =p_{i}-\frac{a n^{c}}{(r q(n)+1) n^{c}-n^{c}}=p_{i}-\frac{a n^{c}}{r q(n) n^{c}} \geq p_{i}-\frac{1}{q(n)} .
\end{aligned}
$$

Lemma 3.4. For all $a, b, c$, all polynomials $q$ and $w$, all $G \in \mathcal{G}_{a, b, c, n}$, and all sequences sq of length $w(n)$, if the average payoff vector of playing $s q$ is $p$, then for all $\delta \leq 1 / f(n)$, where $f(n)=(a-b) w(n) q(n)$, if $s q$ is played infinitely often, player i's payoff in $G^{\infty}(\delta)$ is at least $p_{i}-1 / q(n)$.

Proof. Suppose that $s q=\left(a_{0}, \ldots, a_{w(n)-1}\right)$, and let $v_{i}$ be $i$ 's payoff from $s q^{\infty}$ in $G^{\infty}(\delta)$. Then

$$
\begin{aligned}
v_{i} & =\delta \sum_{t=0}^{\infty}(1-\delta)^{t w(n)} \sum_{k=0}^{w(n)-1} u\left(a_{k}\right)(1-\delta)^{k} \\
& =p_{i}+\delta \sum_{t=0}^{\infty}(1-\delta)^{t w(n)} \sum_{k=0}^{w(n)-1}\left(u\left(a_{k}\right)-p_{i}\right)(1-\delta)^{k} .
\end{aligned}
$$

We want to bound the loss from the second part of the sum. Notice that this is a discounted sum of a sequence whose average payoff is 0 . Call this sequence $s q^{\prime}$. Observe that, because of the discounting, in the worst case, $i$ gets all of his negative payoff in the first round of $s q^{\prime}$ and all his positive payoffs in the last round. Thus, we can bound the discounted average payoff by analyzing this case. Let the sum of $i$ 's negative payoffs in $s q^{\prime}$ be $P_{\text {neg }}$, which means that the sum of $i$ 's positive payoffs must be $-P_{n e g}$. Let $r=a-b$, let $v_{i}^{\prime}=\min _{\vec{a} \in A}\left(u_{i}(\vec{a})-p_{i}\right) \geq-r$, and let $f(n)=r w(n) q(n)$, 
as in the statement of the lemma. So, if $\delta \leq 1 / f(n)$, player $i^{\prime} s$ average discounted payoff in the game is at least

$$
\begin{aligned}
v_{i} & \geq p_{i}+\delta \sum_{t=0}^{\infty} P_{n e g}(1-\delta)^{w(n) t}+\left(-P_{n e g}\right)(1-\delta)^{w(n)(t+1)-1} \\
& =p_{i}+\delta\left(P_{n e g}+\left(-P_{n e g}\right)(1-\delta)^{w(n)-1}\right) \sum_{t=0}^{\infty}(1-\delta)^{w(n) t} \\
& =p_{i}+\delta\left(P_{n e g}+\left(-P_{n e g}\right)(1-\delta)^{w(n)-1}\right) \frac{1}{1-(1-\delta)^{w(n)}} \\
& =p_{i}+P_{n e g} \delta \frac{1-(1-\delta)^{w(n)-1}}{\left(1-(1-\delta)^{w(n)}\right)} \geq p_{i}+\delta P_{n e g} \geq p_{i}+\frac{P_{n e g}}{f(n)} \geq p_{i}+\frac{v_{i}^{\prime} w(n)}{f(n)}=p_{i}-1 / q(n) .
\end{aligned}
$$

The next lemma shows that, for every inverse polynomial, if we "cut off" the game after some appropriately large polynomial $p$ number of rounds (and compute the discounted utility for the finitely repeated game considering only $p(n)$ repetitions), each player's utility in the finitely repeated game is negligibly close to his utility in the infinitely repeated game - that is, the finitely repeated game is a "good" approximation of the infinitely repeated game.

Lemma 3.5. For all $a, b, c$, all polynomials $q$, all $n$, all games $G \in \mathcal{G}_{a, b, c, n}$, all $0<\delta<1$, all strategy profiles $\vec{M}$, and all players $i$, $i$ 's expected utility $p_{i}[\vec{M}]$ in game $G^{\lceil n / \delta\rceil}(\delta)$ and $p_{i}[\vec{M}]$ in game $G^{\infty}(\delta)$ differ by at most a/e $e^{n}$.

Proof. Let $p_{i}^{t}(\vec{M})$ denote player $i$ 's expected utility if the players are playing $\vec{M}$ and the game ends at round $t$. Recall that $(1-\delta)^{1 / \delta} \leq 1 / e$.

$$
\begin{aligned}
& p_{i}^{\infty}(\vec{M})-p_{i}^{\lceil n / \delta\rceil}(\vec{M}) \\
= & \delta \sum_{t=0}^{\infty}(1-\delta)^{t} \sum_{h \in H^{t}, \vec{a} \in A} \rho_{\vec{M}}^{t+1}(h \cdot \vec{a})\left[u_{i}(\vec{a})\right]-\delta \sum_{t=0}^{\lceil n / \delta\rceil}(1-\delta)^{t} \sum_{h \in H^{t}, \vec{a} \in A} \rho_{\vec{M}}^{t+1}(h \cdot \vec{a})\left[u_{i}(\vec{a})\right] \\
= & \delta \sum_{t=\lceil n / \delta\rceil+1}^{\infty}(1-\delta)^{t} \sum_{h \in H^{t}, \vec{a} \in A} \rho_{\vec{M}}^{t+1}(h \cdot \vec{a})\left[u_{i}(\vec{a})\right] \\
\leq & \delta \sum_{t=\lceil n / \delta\rceil}^{\infty}(1-\delta)^{t} a \\
= & \delta(1-\delta)^{\lceil n / \delta\rceil} \sum_{t=0}^{\infty}(1-\delta)^{t} a=\delta(1-\delta)^{\lceil n / \delta\rceil} \frac{a}{\delta} \leq \frac{a}{e^{n}} .
\end{aligned}
$$

\subsubsection{The $\epsilon$-NE strategy and the algorithm}

Let $A_{i}^{0} \subset A_{i}$ be a non-empty set and let $A_{i}^{1}=A_{i} \backslash A_{i}^{0} 6$ A player can broadcast an $m$-bit string by using his actions for $m$ rounds, by treating actions from $A_{i}^{0}$ as 0 and actions from $A_{i}^{1}$ as 1 . Given a polynomial $\phi$ (with natural coefficients), let (Gen, Enc, Dec) be a multi-message multi-key secure $\phi$-bit, if the security parameter is $k$, the length of an encrypted message is $z(k)$ for some polynomial $z$. Let $s q=\left(s_{1}, s_{2} \ldots, s_{m}\right)$ be a fixed sequence of action profiles. Fix a polynomial-time pseudorandom function ensemble $\left\{P S_{s}: s \in\{0,1\}^{*}\right\}$. For a game $G$ such that $|G|=n$, consider the strategy $\sigma^{N E}$ for player $i$ in $G^{\infty}(\delta)$ that has the following three phases. Phase 1 explains what to do if no deviation occurs: play $s q$. Phase 2 gives the preliminaries of what to do if a deviation does occur: roughly, compute a random seed that is shared with all the non-deviating players. Phase 3 explains how to use the random seed to produce a correlated punishment strategy that punishes the deviating player. Formally let $\vec{M}^{\sigma^{N E}}$ be the TMs that implement the following strategy:

\footnotetext{
${ }^{6}$ We assume that each player has at least two actions in $G$. This assumption is without loss of generality-we can essentially ignore players for whom it does not hold.
} 
1. Play according to $s q$ (with wraparound) as long as all players played according to $s q$ in the previous round.

2. After detecting a deviation by player $j \neq i$ in round $t_{0}: 7$

(a) Generate a pair $\left(p k_{i}, s k_{i}\right)$ using Gen $\left(1^{n}\right)$. Store $s k_{i}$ in memory and use the next $l(n)$ rounds to broadcast $p k_{i}$, as discussed above.

(b) If $i=j+1$ (with wraparound), player $i$ does the following:

- $i$ records $p k_{j^{\prime}}$ for all players $j^{\prime} \notin\{i, j\}$;

- $i$ generates a random $n$-bit seed seed;

- for each player $j^{\prime} \notin\{i, j\}, i$ computes $m=E n c_{p k_{j^{\prime}}}$ (seed), and uses the next $(c-2) z(n)$ rounds to communicate these strings to the players other than $i$ and $j$ (in some predefined order).

(c) If $i \neq j+1$, player $i$ does the following:

- $i$ records the actions played by $j+1$ at time slots designated for $i$ to retrieve $\operatorname{Enc}_{P k_{i}}($ seed);

- $i$ decrypts to obtain seed, using Dec and $s k_{i}$.

3. Phase 2 ends after $\phi(n)+(c-2) z(n)$ rounds. The players other than $j$ then compute $P S_{\text {seed }}(t)$ and use it to determine which action profile to play according to the distribution defined by a fixed (correlated) punishment strategy against $j$.

Note that if the players other than $j$ had played a punishment strategy against $j$, then $j$ would get his minimax payoff of 0 . What the players other than $j$ are actually doing is playing an approximation to a punishment strategy in two senses: first they are using a psuedorandom function to generate the randomness, which means that they are not quite playing according to the actual punishment strategy. Also, $j$ might be able to guess which pure strategy profile they are actually playing at each round, and so do better than his minimax value. As we now show, $j$ 's expected gain during the punishment phase is negligible.

Lemma 3.6. For all $a, b$, $c$, all polynomials $t$ and $f$, all $n$, and all games $G \in \mathcal{G}_{a, b, c, n}$, in $G^{\infty}(1 / f(n))$, if the players other than $j$ play $\vec{M}_{-j}^{\sigma^{N E}}$, then if $j$ deviates at round $t(n)$, $j$ 's expected payoff during the punishment phase is negligible.

Proof. Since we want to show $j$ 's expected payoff during the punishment phase (phase (3) only) is negligible, it suffices to consider only polynomially many rounds of playing phase (3) (more precisely, at most $n f(n)$ rounds); by Lemma 3.5. any payoff beyond then is guaranteed to be negligible due to the discounting.

We construct three variants of the strategy $\vec{M}_{-j}^{\sigma^{N E}}$, that vary in phases (2) and (3). We can think of these variants as interpolating between the strategy above and the use of true randomness. (These variants assume an oracle that provides appropriate information; these variants are used only to make the claims precise.)

H1 In phase (2), the punishing players send their public keys to $j+1$. For each player $j^{\prime}$ not being punished, player $j+1$ then encrypts the seed 0 using $\left(j^{\prime}\right)$ 's public key, and then sends the encrypted key to $j^{\prime}$. In phase (3), the punishing players get the output of a truly random function (from an oracle), and use it to play the true punishment strategy. (In this case, phase (2) can be eliminated.)

\footnotetext{
${ }^{7}$ If more than one player deviates while playing $s q$, the players punish the one with the smaller index. The punished player plays his best response to what the other players are doing in this phase.
} 
H2 In phase (2), the punishing players send their public keys to $j+1$. For each player $j^{\prime}$ not being punished, player $j+1$ encrypts the seed 0 using $\left(j^{\prime}\right)$ 's public key, and then sends the encrypted key to $j^{\prime}$. In phase (3), the punishing players get a joint random seed seed (from an oracle) and use the outputs of $P S_{\text {seed }}$ to decide which strategy profile to play in each round. (Again, in this case, phase (2) can be eliminated.)

H3 In phase (2), the punishing players send their public keys to $j+1$. Player $j+1$ chooses a random seed seed and, for each player $j^{\prime}$ not being punished, $j+1$ encrypts seed using $\left(j^{\prime}\right)$ 's public key, and then sends the encrypted key to $j^{\prime}$. In phase (3), the punishing players use the outputs of $P S_{\text {seed }}$ to decide which strategy profile to play in each round.

It is obvious that in $H 1, j$ 's expected payoff is negligible. (Actually, there is a slight subtlety here. As we observed above, using linear programming, we can compute a strategy that gives the correlated minimax, which gives $j$ an expected payoff of 0 . To actually implement this correlated minimax, the players need to sample according to the minimax distribution. They cannot necessarily do this exactly (for example, $1 / 3$ can't be computed exactly using random bits). However, given $n$, the distribution can be discretized to the closest rational number of the form $m / 2^{n}$ using at most $n$ random bits. Using such a discretized distribution, the players other than $j$ can ensure that $j$ gets only a negligible payoff.)

We now claim that in $H 2$, $j$ 's expected payoff during the punishment phase is negligible. Assume for contradiction that a player playing $H 2$ has a non-negligible payoff $\mu(n)$ for all $n$ (i.e., there exists some polynomial $g(\cdot)$ such that $\mu(n) \geq 1 / g(n)$ for infinitely many $n$.). Let $h(n)=n(a-b)^{2}(1 / \mu(n))^{2}$. We claim that if $j$ 's expected payoff is non-negligible, then we can distinguish $h(n)$ instances of the $\mathrm{PRF}\left\{P S_{s}: s \in\{0,1\}^{n}\right\}$ with independently generated random seeds, from $h(n)$ independent truly random functions, contradicting the multi-instance security of the PRF $P S$.

More precisely, we construct a distinguisher $D$ that, given $1^{n}$ and oracle access to a set of functions $f^{1}, f^{2}, \ldots, f^{h(n)}$, proceeds as follows. It simulates $H_{2}$ (it gets the description of the machines to play as its non-uniform advice) $h(n)$ times where in iteration $i^{\prime}$, it uses the function $f^{i^{\prime}}$ as the randomization source of the correlated punishment strategy. $D$ then computes the average payoff of player $j$ in the $h(n)$ runs, and outputs 1 if this average exceeds $\mu(n) / 2$. Note that if the functions $f^{1}, f^{2}, \ldots, f^{h(n)}$ are truly independent random functions, then $D$ perfectly simulates $H_{1}$ and thus, in each iteration $i^{\prime}$, the expected payoff of player $j$ (during the punishment phase) is negligible. On the other hand, if the functions $f^{1}, f^{2}, \ldots, f^{h(n)}$ are $h(n)$ independent randomly chosen instances of the PRF $\left\{P S_{s}: s \in\{0,1\}^{n}\right\}$, then $D$ perfectly simulates $H_{2}$, and thus, in each iteration $i^{\prime}$, the expected payoff of player $j$ (during the punishment phase) is at least $\mu(n)$.

By Hoeffding's inequality Hoeffding 1963, given $m$ random variables $X_{1}, \ldots, X_{m}$ all of which take on values in an interval of size $c^{\prime}, p(|\bar{X}-E(\bar{X})| \geq r) \leq 2 \exp \left(-\frac{2 m r^{2}}{c^{\prime 2}}\right)$. Since, in this setting, the range of the random variables is an interval of size $a-b$, the probability that $D$ outputs 1 when the function are truly independent is at most $2 / e^{n}$, while the probability that $D$ outputs 1 when the functions are independent randomly chosen instances of the $\operatorname{PRF}\left\{P S_{s}: s \in\{0,1\}^{n}\right\}$ is at least $1-2 / e^{n}$. This, in turn, means that the difference between them is not negligible, which is a contradiction. Thus, $j$ 's expected payoff in $H 2$ must be negligible.

We now claim that in $H 3$, player $j$ 's expected payoff during the punishment phase is also negligible. Indeed, if $j$ can get a non-negligible payoff, then we can break the multi-message multikey secure encryption scheme.

Again, assume for contradiction that the punished player's expected payoff in the punishment phase is a non-negligible function $\mu(n)$ for all $n$. We can build a distinguisher $A=\left(A_{1}, A_{2}\right)$ (which also gets the description of the machines to play as its non-uniform advice) to distinguish $\left\{\operatorname{IND}_{-M_{U L T}}^{\Pi}(A, n, h, c)\right\}_{n}$ and $\left\{\operatorname{IND}_{0} \operatorname{MULT}_{1}^{\Pi}(A, n, h, c)\right\}_{n}$ (where we abuse notation and identify 
$c$ with the constant polynomial that always returns $c)$. Given $n, A_{1}$ randomly selects $h(n)$ messages $r_{1}, \ldots, r_{h(n)}$ and outputs $\left(0, \ldots, 0, r_{1}, \ldots, r_{h(n)},\left(p k_{1}, \ldots, p k_{c}\right)\right) . A_{2}$ splits its input into pieces. The first piece contains the first $c$ encryptions in $\mathcal{C}$ (i.e., the $c$ encryptions of the first message chosen, according to the $c$ different encryption functions), the second the next $c$ encryptions and so on. Notice that each piece consists of $c$ different encryptions of the same message in both cases. It can also simulate phase (1) by following the strategy for $t$ rounds. It then uses each piece, along with the public keys, to simulate the communication in phase (2). For piece $j$ it uses $r_{j}$ as the seed of the PRF in phase (3). It repeats this experiment for all the different pieces of the input, for a total of $h(n)$ times, and outputs 1 if the punished player's average payoff over all experiments using its strategy is more than $\mu(n) / 2$.

Note that if $b=1$, player $j$ faces $H 3$ (i.e., the distributions over runs when $b=1$ is identical to the distribution over runs with $\mathrm{H} 3$, since in both cases the seed is chosen at random and the corresponding messages are selected the same way), so player j's expected payoff in the punishment phase is $\mu(n)$. Thus, by Hoeffding's inequality the probability that player j's average payoff in the punishment phase is more then $\mu(n) / 2$ is $1-2 / e^{n}$, so $A_{2}$ outputs 1 with that probability in the case $b=1$. On the other hand, if $b=0$, then this is just $H_{2}$. We know player $j$ 's expected payoff in the punishment phase in each experiment is no more than negligible in $H_{2}$, so the probability that the average payoff is more than $\mu(n) / 2$ after $h(n)$ rounds, is negligible. This means that there is a non-negligible difference between the probability $A$ outputs 1 when $b=1$ and when $b=0$, which contradicts the assumption that the encryption scheme is multi-message multi-key secure public key secure. Thus, the gain in $H 3$ must be negligible.

$H 3$ is exactly the game that the punished player faces; thus, this shows he can't hope to gain more than a negligible payoff in expectation.

We can now state and prove our main theorem, which says that $\sigma^{N E}$ is an $\epsilon$-NE for all inverse polynomials $\epsilon$ and the can be computed in polynomial time.

Theorem 3.7. For all $a, b, c$, and all polynomials $q$, there is a polynomial $f$ and a polynomial-time algorithm $F$ such that, for all sequences $G_{1}, G_{2}, \ldots$ of games with $G^{j} \in G_{a, b, c, j}$ and for all inverse polynomials $\delta \leq 1 / f$, the sequence of outputs of $F$ given the sequence $G_{1}, G_{2}, \ldots$ of inputs is a $\frac{1}{q}-N E$ for $G_{1}^{\infty}(\delta(1)), G_{2}^{\infty}(\delta(2)), \ldots$

Proof. Given a game $G^{n} \in \mathcal{G}(a, b, c, n)$, the first step of the algorithm is to find a correlated equilibrium $\sigma$ of $G^{n}$. This can be done in polynomial time using linear programming. Since the minimax value of the game is 0 for all players, all players have an expected utility of at least 0 using $\sigma$. Let $r=a-b$. By Lemma 3.3, we can construct a sequence $s q$ of length $w(n)=(3 r n q(n)+1) n^{c}$ that has an average payoff for each player that is at most $1 / 3 q(n)$ less than his payoff using $\sigma$. By Lemma 3.4, it follows that by setting the discount factor $\delta<1 / f^{\prime}(n)$, where $f^{\prime}(n)=3 r w(n) q(n)$, the loss due to discounting is also at most $1 / 3 q(n)$. We can also find a punishment strategy against each player in polynomial time, using linear programming.

We can now compute the strategy $\vec{M}^{\sigma^{N E}}$ described earlier that uses the sequence $s q$ and the punishment strategies. Let $\vec{\sigma}_{n}^{*}$ be this strategy when given $g_{n}$ as input. Let $m(n)$ be the length of phase (2). (Note that $m(n)$ is a polynomial that depends only on the choice of encryption schemethat is, it depends on $l$, where an $l$-bit public-key encryption scheme is used, and on $z$, where $z(k)$ is the length of encrypted messages.) Let

$$
f(n)=\max \left(3 q(n)(m(n) a+1), f^{\prime}(n)\right) .
$$

Notice that $f$ is independent of the actual game as required.

We now show that $\vec{\sigma}_{1}^{*}, \ldots$ as defined above is a $(1 / q)$-NE. If in game $G^{n}$ a player follows $\sigma_{n}^{*}$, he gets at least $-2 / 3 q(n)$. Suppose that player $j$ defects at round $t$; that is, that he plays according 
to $\sigma_{n}^{*}$ until round $t$, and then defects. By Lemma 3.5 if $t>\frac{n}{\delta(n)}$, then any gain from defection is negligible, so there exists some $n_{1}$ such that, for all $n>n_{1}$, a defection in round $t$ cannot result in the player gaining more than $\frac{1}{q(n)}$. If player $j$ defects at round $t \leq \frac{n}{\delta(n)}$, he gets at most $a$ for the duration of phase (2), which is at most $m(n)$ rounds, and then, by Lemma 3.6, gains only a negligible amount, say $\epsilon_{n e g}(n)$ (which may depend on the sequence of deviations), in phase (3). Let $u_{i}^{n}$ be the payoff of player $i$ in game $G^{n}$ of the sequence. It suffices to show that

$$
\begin{array}{r}
\delta(n)\left(\sum_{k=0}^{t} u_{i}^{n}\left(a_{k}\right)(1-\delta(n))^{k}+\sum_{k=0}^{m(n)} a(1-\delta(n))^{k+t}+(1-\delta(n))^{t+m(n)} \epsilon_{n e g}(n)\right)-1 / q(n) \\
\leq \delta(n)\left(\sum_{k=0}^{t} u_{i}^{n}\left(a_{k}\right)(1-\delta(n))^{k}+\sum_{k=t}^{\infty} u_{i}^{n}\left(a_{k}\right)(1-\delta(n))^{k}\right) .
\end{array}
$$

By deleting the common terms from both side, rearranging, and noticing that $(1-\delta(n))^{m(n)} \epsilon_{n e g(n)} \leq \epsilon_{n e g(n)}$, it follows that it suffices to show

$$
\delta(n)(1-\delta(n))^{t}\left(\sum_{k=0}^{m(n)} a(1-\delta(n))^{k}+\epsilon_{n e g}(n)\right)-\frac{1}{q(n)} \leq \delta(n)(1-\delta(n))^{t}\left(\sum_{k=0}^{\infty} u_{i}^{n}\left(a_{k+t}\right)(1-\delta(n))^{k}\right) .
$$

We divide both sides of the equation by $(1-\delta(n))^{t}$. No matter at what step of the sequence the defection happens, the future expected discounted payoff from that point on is still at least $-2 / 3 q(n)$, as our bound applies for the worst sequence for a player, and we assumed that in equilibrium all players get at least 0 . It follows that we need to show

$$
\delta(n)\left(\sum_{k=0}^{m(n)} a(1-\delta(n))^{k}+\epsilon_{n e g}(n)\right)-\frac{1}{q(n)(1-\delta(n))^{t}} \leq-\frac{2}{3 q(n)} .
$$

Since $\epsilon_{\text {neg }}$ is negligible for all deviations, it follows that, for all sequences of deviations, there exists $n_{0}$ such that $\epsilon_{n e g}(n)<1$ for all $n \geq n_{0}$. For $n \geq n_{0}$,

$$
\begin{aligned}
& \delta(n)\left(\sum_{k=0}^{m(n)} a(1-\delta(n))^{k}+\epsilon_{n e g}(n)\right)-\frac{1}{q(n)(1-\delta(n))^{t}} \\
\leq & \delta(n)\left(m(n) a+\epsilon_{n e g}(n)\right)-\frac{1}{q(n)} \\
\leq & \frac{m(n) a+\epsilon_{n e g}(n)}{f(n)}-\frac{1}{q(n)} \\
\leq & \frac{m(n) a+\epsilon_{n e g}(n)}{3 q(n)(m(n) a+1)}-\frac{1}{q(n)} \\
\leq & \frac{1}{3 q(n)}-\frac{1}{q(n)} \\
= & -\frac{2}{3 q(n)} .
\end{aligned}
$$

This shows that there is no deviating strategy that can result in the player gaining more than $\frac{1}{q(n)}$ in $G^{n}$ for $n>\max \left\{n_{0}, n_{1}\right\}$.

\subsection{Dealing with a variable number of players}

Up to now, we have assumed, just as in Borgs et al. [2010], that the number of players in the game is a fixed constant $(\geq 3)$.

What happens if the number of players in the game is part of the input? In general, describing the players' utilities in such a game takes space exponential in the number of players (since there are exponentially many strategy profiles). Thus, to get interesting computational results, we consider games that can be represented succinctly. 
Graphical games Kearns, Littman, and Singh 2001 of degree $d$ are games that can be represented by a graph in which each player is a node in the graph, and the utility of a player is a function of only his action and the actions of the players to which he is connected by an edge. The maximum degree of a node is assumed to be at most $d$. This means a player's punishment strategy depends only on the actions of at most $d$ players.

Definition 3.8. Let $\mathcal{G}_{a, b, d, n, m}^{\prime}$ be the set of all graphical games with degree at most $d$, at most $m$ players and at most $n$ actions per player, integral payoffs 8 maximum payoff a, and minimum payoff $b$.

The following corollary then follows from our theorem, the fact that a correlated equilibrium with polynomial sized-support can be computed in polynomial time [Jiang and Leyton-Brown 2011], and the observation that we can easily compute a correlated minimax strategy that depends only on the action of at most $d$ players.

Corollary 3.9. For all $a, b, d$, and all polynomials $q$, there is a polynomial $f$ and a polynomialtime algorithm $F$ such that, for all sequences $G_{1}, G_{2}, \ldots$ of games with $G^{j} \in G_{a, b, d, j, j}$ and for all inverse polynomials $\delta \leq 1 / f$, the sequence of outputs of $F$ given the sequence $G_{1}, G_{2}, \ldots$ of inputs is a $\frac{1}{q}$-equilibrium for $G_{1}^{\infty}(\delta(1)), G_{2}^{\infty}(\delta(2)), \ldots$

\section{Computational subgame-perfect equilibrium}

\subsection{Motivation and Definition}

In this section we would like to define a notion similar to subgame-perfect equilibrium, where for all histories $h$ in the game tree (even ones not on the equilibrium path), playing $\vec{\sigma}$ restricted to the subtree starting at $h$ forms a NE. This means that a player does not have any incentive to deviate, no matter where he finds himself in the game tree.

As we suggested in the introduction, there are a number of issues that need to be addressed in formalizing this intuition in our computational setting. First, since we consider stateful TMs, there is more to a descriptione of a situation than just the history; we need to know the memory state of the TM. That is, if we take a history to be just a sequence of actions, then the analogue of history for us is really a pair $(h, \vec{m})$ consisting of a sequence $h$ of actions, and a profile of memory states, one for each player. Thus, to be a computational subgame-perfect equilibrium the strategies should be a NE at every history and no matter what the memory states are.

Another point of view is to say that the players do not in fact have perfect information in our setting, since we allow the TMs to have memory that is not observed by the other players, and thus the game should be understood as a game of imperfect information. In a given history $h$ where $i$ moves, $i$ 's information set consists of all situations where the history is $h$ and the states of memory of the other players are arbitrary. While subgame-perfect equilibrium extends to imperfect information games it usually doesn't have much bite (see Kreps and Wilson 1982 for a discussion on this point). For the games that we consider, subgame-perfect equilibrium typically reduces to NE. An arguably more natural generalization of subgame-perfect equilibrium in imperfect-information games would require that if an information set for player $i$ off the equilibrium path is reached, then player $i$ 's strategy is a best response to the other players' strategies no matter how that information set is reached. This is quite a strong requirement. (see Osborne and Rubinstein 1994] [pp. 219-221] for a discussion of this issue); such equilibria do not in general exist in games of imperfect information.

Instead, in games of imperfect information, the solution concept most commonly used is sequential equilibrium Kreps and Wilson 1982. A sequential equilibrium is a pair $(\vec{\sigma}, \mu)$ consisting of a

\footnotetext{
${ }^{8}$ Again, our result also holds for rational payoffs, except then the size of the game needs to take into account the bits needed to represent the payoffs.
} 
strategy profile $\vec{\sigma}$ and a belief system $\mu$, where $\mu$ associates with each information set $I$ a probability $\mu(I)$ on the nodes in $I$. Intuitively, if $I$ is an information set for player $i, \mu(I)$ describes $i$ 's beliefs about the likelihood of being in each of the nodes in $I$. Then $(\vec{\sigma}, \mu)$ is a sequential equilibrium if, for each player $i$ and each information set $I$ for player $i, \sigma_{i}$ is a best response to $\vec{\sigma}_{-i}$ given $i$ 's beliefs $\mu(I)$. However, a common criticism of this solution concept is that it is unclear what these beliefs should be and how players create these beliefs. Instead, our notion of computational subgame-perfection can be viewed as a strong version of a sequential equilibrium, where, for each player $i$ and each information set $I$ for $i, \sigma_{i}$ is a best response to $\vec{\sigma}_{-i}$ conditional on reaching $I$ (up to $\epsilon$ ) no matter what $i$ 's beliefs are at $I$.

As a deviating TM can change its memory state in arbitrary ways, when we argue that a strategy profile is an $\epsilon$-NE at a history, we must also consider all possible states that the TM might start with at that history. Since there exists a deviation that just rewrites the memory in the round just before the history we are considering, any memory state (of polynomial length) is possible. Thus, in the computational setting, we require that the TM's strategies are an $\epsilon$-NE at every history, no matter what the states of the TMs are at that history. This solution concept is in the spirit of subgame-perfect equilibrium, as we require that the strategies are a NE after every possible deviation, although the player might not have complete information as to what the deviation is.

Intuitively, a profile $\vec{M}$ of TMs is a computational subgame-perfect equilibrium if for all players $i$, all histories $h$ where $i$ moves, and all memory profiles $\vec{m}$ of the players, there is no polynomial-time TM $\bar{M}$ such that player $i$ can gain more than $\epsilon$ by switching from $M_{i}$ to $\bar{M}$. To make it precise, we must again consider an infinite sequence of games of increasing size (just as we do for NE, although this definition is more complicated since we must consider memory states).

For a memory state $m$ and a TM $M$ let $M(m)$, stand for running $M$ with initial memory state $m$. We use $\vec{M}(\vec{m})$ to denote $\left(M_{1}\left(m_{1}\right), \ldots, M_{c}\left(m_{c}\right)\right)$. Let $p_{i}^{G, \delta}(\vec{M})$ denote player i's payoff in $G^{\infty}(\delta)$ when $\vec{M}$ is played.

Definition 4.1. An infinite sequence of strategy profiles $\vec{M}^{1}, \vec{M}^{2}, \ldots$, where $\vec{M}^{k}=\left(M_{1}^{k}, \ldots, M_{c}^{k}\right)$, is a computational subgame-perfect $\epsilon$-equilibrium of an infinite sequence $G_{1}^{\infty}, G_{2}^{\infty}, \ldots$ of repeated games where the size of $G_{k}$ is $k$, if, for all players $i \in[c]$, all sequences $h_{1} \in H_{G_{1}^{\infty}}, h_{2} \in H_{G_{2}^{\infty}}, \ldots$ of histories, all sequences $\vec{m}^{1}, \vec{m}^{2}, \ldots$ of polynomial-length memory-state profiles, where $\vec{m}^{k}=\left(m_{1}^{k}, \ldots, m_{c}^{k}\right)$, and all non-uniform PPT adversaries $\bar{M}$ (polynomial in $k$ and $t$, as discussed above), there exists $k_{0}$ such that, for all $k \geq k_{0}$,

$$
p_{i}^{G_{k}^{\infty}\left(h_{k}\right), \delta}\left(\bar{M}\left(m_{i}^{k}\right), \vec{M}_{-i}^{k}\left(\vec{m}_{-i}^{k}\right)\right) \leq p_{i}^{G_{k}^{\infty}\left(h_{k}\right), \delta}\left(\vec{M}^{k}\left(\vec{m}^{k}\right)\right)+\epsilon(k) .
$$

\subsection{Computing a subgame-perfect $\epsilon$-NE}

For a game $G$ such that $|G|=n$, and a polynomial $\ell$, consider the following strategy $\sigma^{N E, \ell}$, and let $\vec{M}^{\sigma^{N E, \ell}}$ be the TMs that implement this strategy. This strategy is similar in spirit to that proposed in Section 3.2.2 indeed, the first two phases are identical. The key difference is that the punishment phase is played for only $\ell(n)$ rounds. After that, players return to phase 1 . As we show, this limited punishment is effective since it is not played long enough to make it an empty threat (if $\ell$ is chosen appropriately). Phase 4 takes care of one minor issue: The fact that we can start in any memory state means that a player might be called on to do something that, in fact, he cannot do (because he doesn't have the information required to do it). For example, he might be called upon to play the correlated punishment strategy in a state where he has forgotten the random seed, so he cannot play it. In this case, a default action is played. Note that his was not an issue in the analysis of NE.

1. Play according to $s q$ (with wraparound) as long as all players played according to $s q$ in the previous round. 
2. After detecting a deviation by player $j \neq i$ in round $t_{0} 9$

(a) Generate a pair $\left(p k_{i}, s k_{i}\right)$ using Gen $\left(1^{n}\right)$. Store $s k_{i}$ in memory and use the next $l(n)$ rounds to broadcast $p k_{i}$, as discussed above.

(b) If $i=j+1$ (with wraparound), player $i$ does the following:

- $i$ records $p k_{j^{\prime}}$ for all players $j^{\prime} \notin\{i, j\}$;

- $i$ generates a random $n$-bit seed seed;

- for each player $j^{\prime} \notin\{i, j\}, i$ computes $m=E n c_{p k_{j^{\prime}}}($ seed $)$, and uses the next $(c-2) z(n)$ rounds to communicate these strings to the players other than $i$ and $j$ (in some predefined order).

(c) If $i \neq j+1$, player $i$ does the following:

- $i$ records the actions played by $j+1$ at time slots designated for $i$ to retrieve $E c_{P k_{i}}($ seed $)$;

- $i$ decrypts to obtain seed, using $D e c$ and $s k_{i}$.

3. Phase 2 ends after $\phi(n)+(c-2) z(n)$ rounds. The players other than $j$ then compute $P S_{\text {seed }}(t)$ and use it to determine which action profile to play according to the distribution defined by a fixed (correlated) punishment strategy against $j$. Player $j$ plays his best response to the correlated punishment strategy throughout this phase. After $\ell(n)$ rounds, they return to phase 1, playing the sequence $s q$ from the point at which the deviation occurred (which can easily be inferred from the history).

4. If at any point less than or equal to $\phi(n)+(c-2) z(n)$ time steps from the last deviation from phase 1 the situation is incompatible with phase 2 as described above (perhaps because further deviations have occurred), or at any point between $\phi(n)+(c-2) z(n)$ and $\phi(n)+(c-$ $2) z(n)+\ell(n)$ steps since the last deviation from phase 1 the situation is incompatible with phase 3 as described above, play a fixed action for the number of rounds left to complete phases 2 and 3 (i.e., up to $\phi(n)+(c-2) z(n)+\ell(n)$ steps from the last deviation from phase 1). Then return to phase 1.

Note that with this strategy a deviation made during the punishment phase is not punished. Phase 2 and 3 are always played to their full length (which is fixed and predefined by $\ell$ and $z$ ). We say that a history $h$ is a phase 1 history if it is a history where an honest player should play according to $s q$. History $h$ is a phase 2 history if it is a history where at most $\phi(n)+(c-2) z(n)$ rounds have passed since the last deviation from phase $1 ; h$ is a phase 3 history if more than $\phi(n)+(c-2) z(n)$ but at most $\phi(n)+(c-2) z(n)+\ell(n)$ rounds have passed since the last deviation from phase 1 . No matter what happens in phase 2 and 3 , a history in which exactly $\phi(n)+(c-2) z(n)+\ell(n)$ round have passed since the last deviation from phase 1 is also a phase 1 history (even if the players deviate from phase 2 and 3 in arbitrary ways). Thus, no matter how many deviations occur, we can uniquely identify the phase of each round.

We next show that by selecting the right parameters, these strategies are easy to compute and are a subgame-perfect $\epsilon$-equilibrium for all inverse polynomials $\epsilon$.

Definition 4.2. Let $\mathcal{G}_{a, b, c, n}$ be the set of all games with c players, at most $n$ actions per player, integral payoffs, maximum payoff $a$, and minimum payoff $b$.

\footnotetext{
${ }^{9}$ Again, if more than one player deviates while playing $s q$, the players punish the one with the smaller index. The punished player plays his best response to what the other players are doing in this phase.
} 
We first show that for any strategy that deviates while phase 1 is played, there is a strategy whose payoff is at least as good and either does not deviate in the first polynomially many rounds, or after its first deviation, deviates every time phase 1 is played. (Recall that after every deviation in phase 1 , the other players play the punishment phase for $\ell(n)$ rounds and then play phase 1 again.)

We do this by showing that if player $i$ has a profitable deviation at some round $t$ of phase 1 , then it must be the case that every time this round of phase 1 is played, $i$ has a profitable deviation there. (That is, the strategy of deviating every time this round of phase 1 is played is at least as good as a strategy where player $i$ correlates his plays in different instantiations of phase 1.) While this is trivial in traditional game-theoretic analyses, naively applying it in the computational setting does not necessarily work. It requires us to formally show how we reduce a polynomial time TM $M$ to a different TM $M^{\prime}$ of the desired form without blowing up the running time and size of the TM.

For a game $G$, let $H_{G^{\infty}}^{1, n}$ be the set of histories $h$ of $G^{\infty}$ of length at most $n f(n)$ such that at (the last node of) $h, \sigma^{N E, \ell}$ is in phase 1 . Let $R(M)$ be the polynomial that bounds the running time of TM $M$.

Definition 4.3. Given a game $G$, a deterministic $T M M$ is said to be $(G, f, n)$-well-behaved if, when $\left(M, \sigma_{-i}^{N E, \ell}\right)$ is played, then either $M$ does not deviate for the first $n f(n)$ rounds or, after $M$ first deviates, $M$ continues to deviate from sq every time phase 1 is played in the next $n f(n)$ rounds.

Lemma 4.4. For all $a, b, c$, and all polynomials $f$, there exists a polynomial $g$ such that for all $n$, all games $G \in \mathcal{G}_{a, b, c, n}$, all $h \in H_{G^{\infty}}^{1, n}$, all players $i$, and all TMs $M$, there exists a $(G(h), f, n)$ well-behaved TM M' such that $p_{i}^{G^{h}, 1 / f(n)}\left(M^{\prime}, \vec{M}_{-i}^{\sigma^{N E, \ell}}\right) \geq p_{i}^{G^{h}, 1 / f(n)}\left(M, \vec{M}_{-i}^{\sigma^{N E, \ell}}\right)$, and $R\left(M^{\prime}\right),\left|M^{\prime}\right| \leq$ $g(R(M))$.

Proof. Suppose that we are given $G \in \mathcal{G}_{a, b, c, n}, h \in H_{G^{\infty}}^{1, n, f}$, and a TM $M$. We can assume without loss of generality that $M$ is deterministic (we can always just use the best random tape). If $M$ does not deviate in the first $n f(n)$ rounds of $G(h)^{\infty}$ then $M^{\prime}$ is just $M$, and we are done. Otherwise, we construct a sequence of TMs starting with $M$ that are, in a precise sense, more and more well behaved, until eventually we get the desired TM $M^{\prime}$.

For $t_{1}<t_{2}$, say that $M$ is $\left(t_{1}, t_{2}\right)-(G, f, n)$-well-behaved if $M$ does not deviate from $s q$ until round $t_{1}$, and then deviates from $s q$ every time phase 1 is played up to (but not including) round $t_{2}$ (by which we mean there exists some history in which $M$ does not deviate at round $t_{2}$ and this is the shortest such history over all possible random tapes of $\vec{M}_{-i}^{\sigma^{N E, \ell}}$ ). We construct a sequence $M_{1}, M_{2}, \ldots$ of TMs such that (a) $M_{1}=M$, (b) $M_{i}$ is $\left(t_{1}^{i}, t_{2}^{i}\right)-(G, f, n)$-well-behaved, (c) either $t_{1}^{i+1}>t_{i}$ or $t_{1}^{i+1}=t_{1}^{i}$ and $t_{2}^{i+1}>t_{2}^{i}$, and (d) $p_{i}^{G^{h}, 1 / f(n)}\left(M_{i+1}, \vec{M}_{-i}^{\sigma^{N E, \ell}}\right) \geq p_{i}^{G^{h}, 1 / f(n)}\left(M_{i}, \vec{M}_{-i}^{\sigma^{N E, \ell}}\right)$. Note that if $t_{1} \geq n f(n)$ or $t_{2} \geq t_{1}+n f(n)$, then a $\left(t_{1}, t_{2}\right)-(G, f, n)$-well-behaved TM is $(G, f, n)$ well-behaved.

Let $t<n f(n)$ be the first round at which $M$ deviates. (This is well defined since the play up to $t$ is deterministic.) Let the history up to time $t$ be $h^{t}$. If $M$ deviates every time that phase 1 is played for the $n f(n)$ rounds after round $t$, then again we can take $M^{\prime}=M$, and we are done. If not, let $t^{\prime}$ be the first round after $t$ at which phase 1 is played and there exists some history of length $t^{\prime}$ at which $M$ does not deviate. By definition, $M$ is $\left(t, t^{\prime}\right)-(G, f, n)$-well behaved. We take $M_{1}=M$ and $\left(t_{1}^{1}, t_{2}^{1}\right)=\left(t, t^{\prime}\right)$. (Note that since $\vec{M}_{-i}^{\sigma^{N E, \ell}}$ are randomized during phase 2 , the first time after $t$ at which $M$ returns to playing phase 1 and does not deviate may depend on the results of their coin tosses. We take $t^{\prime}$ to be the first time this happens with positive probability.)

Let $s^{h^{*}}$ be $M$ 's memory state at a history $h^{*}$. We assume for ease of exposition that $M$ encodes the history in its memory state. (This can be done, since the memory state at time $t$ is of size polynomial in $t$.) Consider the TM $M^{\prime \prime}$ that acts like $M$ up to round $t$, and copies $M$ 's memory state at that round (i.e., $s^{h^{t}}$ ). $M^{\prime \prime}$ continues to plays like $M$ up to the first round $t^{\prime}$ with $t<t^{\prime}<t+n f(n)$ 
at which $\sigma^{N E, \ell}$ would be about to return to phase 1 and $M$ does not deviate (which means that $M$ plays an action in the sequence $s q$ at round $t^{\prime}$ ). At round $t^{\prime}, M^{\prime \prime}$ sets its state to $s^{h^{t}}$ and simulates $M$ from history $h^{t}$ with states $s^{h(t)}$; so, in particular, $M^{\prime \prime}$ does deviate at time $t^{\prime}$. (Again, the time $t^{\prime}$ may depend on random choices made by $\vec{M}_{-i}^{\sigma^{N E, \ell}}$. We assume that $M^{\prime \prime}$ deviates the first time $M$ is about to play phase 1 after round $t$ and does not deviate, no matter what the outcome of the coin tosses.) This means, in particular, that $M^{\prime \prime}$ deviates at any such $t^{\prime}$. We call $M^{\prime \prime}$ a type 1 deviation from $M$.

If $p_{i}^{G^{h^{t}}, 1 / f(n)}\left(M^{\prime \prime}, \vec{M}_{-i}^{\sigma^{N E, \ell}}\right)>p_{i}^{G^{h^{t}}, 1 / f(n)}\left(M, \vec{M}_{-i}^{\sigma^{N E, \ell}}\right)$, then we take $M_{2}=M^{\prime \prime}$. Note that $t_{1}^{2}=t_{1}^{1}=t$, while $t_{2}^{2}>t_{2}^{1}=t^{\prime}$, since $M^{\prime \prime}$ deviates at $t^{\prime}$. If $p_{i}^{G^{h^{t}}, 1 / f(n)}\left(M^{\prime \prime}, \vec{M}_{-i}^{\sigma^{N E, \ell}}\right)<$ $p_{i}^{G^{h^{t}}, 1 / f(n)}\left(M, \vec{M}_{-i}^{\sigma^{N E, \ell}}\right)$, then there exists some history $h^{*}$ of both $M$ and $M^{\prime \prime}$ such that $t<$ $\left|h^{*}\right|<t+n f(n), M^{\prime \prime}$ deviates at $h^{*}, M$ does not, and $M$ has a better expected payoff than $M^{\prime \prime}$ at $h^{*}$. (This is a history where the type 1 deviation failed to improve the payoff.) Take $M_{2}$ to be the TM that plays like $\vec{M}_{i}^{\sigma^{N E, \ell}}$ up to time $t$, then sets its state to $s^{h^{*}}$, and then plays like $M$ with state $s^{h^{*}}$ in history $h^{*}$. We call $M_{2}$ a type 2 deviation from $M$. Note that $M_{2}$ does not deviate at $h^{t}$ (since $M$ did not deviate at history $h^{*}$ ). Let $\delta^{\prime}=(1-\delta)^{\left|h^{*}\right|-\left|h^{t}\right|}$. Clearly $\delta^{\prime} p_{i}^{G^{h^{t}}, 1 / f(n)}\left(M_{2}, \vec{M}_{-i}^{\sigma^{N E, \ell}}\right)=p_{i}^{G^{h^{*}}, 1 / f(n)}\left(M, \vec{M}_{-i}^{\sigma^{N E, \ell}}\right)$, since $\vec{M}_{-i}^{\sigma^{N E, \ell}}$ acts the same in $G^{h^{t}}$ and $G^{h^{*}}$. Since $M^{\prime \prime}$ plays like $M\left(s^{h_{t}}\right)$ at $h^{*}, p_{i}^{G^{h^{*}}, 1 / f(n)}\left(M^{\prime \prime}, \vec{M}_{-i}^{\sigma^{N E, \ell}}\right)=\delta^{\prime} p_{i}^{G^{h^{t}}, 1 / f(n)}\left(M, \vec{M}_{-i}^{\sigma^{N E, \ell}}\right)$. Combining this with the previous observations, we get that $p_{i}^{G^{h^{t}}, 1 / f(n)}\left(M_{2}, \vec{M}_{-i}^{\sigma^{N E, \ell}}\right) \geq p_{i}^{G^{h^{t}}, 1 / f(n)}\left(M, \vec{M}_{-i}^{\sigma^{N E, \ell}}\right)$. Also note that $t_{1}^{2}>t_{1}^{1}$. This completes the construction of $M_{2}$. We inductively construct $M_{i+1}$, $i=2,3, \ldots$, just as we $\operatorname{did} M_{2}$, letting $M_{i}$ play the role of $M$.

Next observe that, without loss of generality, we can assume that this sequence arises from a sequnce of type 2 deviations, followed by a sequence of type 1 deviations: For let $j_{1}$ be the first point in the sequence at which a type 1 deviation is made. We claim that we can assume without loss of generality that all further deviations are type 1 deviations. By assumption, since $M_{j_{1}}$ gives $i$ higher utility than $M_{j_{1}-1}$, it is better to deviate the first time $M_{j_{1}-1}$ wants to play phase 1 again after an initial deviation. This means that when $M_{j_{1}}$ wants to play phase 1 again after an initial deviation it must be better to deviate again, since the future play of the $\vec{M}_{-i}^{\sigma^{N E, \ell}}$ is the same in both of these situations. This means that once a type 1 deviation occurs, we can assume that all further deviations are type 1 deviations.

Let $M_{j}$ be the first TM in the sequence that is well behaved. (As we observed earlier, there must be such a TM.) Using the fact that the sequence consists of a sequence of type 2 deviations followed by a sequence of type 1 deviations, it is not hard to show that $M_{j}$ can be implemented efficiently. First notice that $M_{j_{1}}$ is a TM that plays like $\vec{M}_{i}^{\sigma^{N E, \ell}}$ until some round, and then plays $M$ starting with its state at a history which is at most $(n f(n))^{2}$ longer than the real history at this point. This is because its initial history becomes longer by at most $n f(n)$ at each round and we iterate this construction at most $n f(n)$ times. This means that its running time is obviously polynomially related to the running time of the original $M$. The same is true of the size of $M_{j_{1}}$, since we need to encode only the state at this initial history and the history at which we switch, which is polynomially related to $R(M)(n)$.

To construct $M_{j}$, we need to modify $M_{j_{1}}$ only slightly, since only type 1 deviations occur. Specifically, we need to know only $t_{j_{1}}^{1}$ and to encode its state at this round. At every history after that, we run $M_{J_{1}}$ (which is essentially running $M$ on a longer history) on a fixed history, with a potential additional step of copying the state. It is easy to see that the resulting TM has running time and size at most $O(R(M))$. 
We now state and prove our theorem, which shows that there exists a polynomial-time algorithm for computing a subgame-perfect $\epsilon$-equilibrium by showing that, for all inverse polynomials $\epsilon$, there exists a polynomial function $\ell$ of $\epsilon$ such that $\sigma^{N E^{*}, \ell}$ is a subgame-perfect $\epsilon$-equilibrium of the game. The main idea of the proof is to show that the players can't gain much from deviating while the sequence is being played, and also that, since the punishment is relatively short, deviating while a player is being punished is also not very profitable.

Theorem 4.5. For all $a, b, c$, and all polynomials $q$, there is a polynomial $f$ and a polynomial-time algorithm $F$ such that, for all sequences $G_{1}, G_{2}, \ldots$ of games with $G^{j} \in G_{a, b, c, j}$ and for all inverse polynomials $\delta \leq 1 / f$, the sequence of outputs of $F$ given the sequence $G_{1}, G_{2}, \ldots$ of inputs is a subgame-perfect $\frac{1}{q}$-equilibrium for $G_{1}^{\infty}(\delta(1)), G_{2}^{\infty}(\delta(2)), \ldots$

Proof. Given a game $G^{n} \in \mathcal{G}(a, b, c, n)$, the algorithm finds a correlated equilibrium $\sigma$ of $G^{n}$, which can be done in polynomial time using linear programming. Each player's expected payoff is at least 0 when playing $\sigma$, since we assumed that the minimax value of the game is 0 . Let $r=a-b$. By Lemma 3.3 and Lemma 3.4, we can construct a sequence $s q$ of length $w(n)=4(r n q(n)+1) n^{c}$ and set $f^{\prime}(n)=4 r w(n) q(n)$, so that if the players play $s q$ infinitely often and $\delta<1 / f^{\prime}(n)$, then all the players get at least $-1 / 2 q(n)$. The correlated punishment strategy against each player can also be found in polynomial time using linear programming.

Let $m(n)$ be the length of phase 2, including the round where the deviation occurred. (Note that $m(n)$ is a polynomial that depends only on the choice of encryption scheme - that is, it depends on $\phi$, where a $\phi$-bit public-key encryption scheme is used, and on $z$, where $z(k)$ is the length of encrypted messages.) Let $\ell(n)=n q(n)(m(n) a+1)$, let $\sigma_{n}^{*}$ be the strategy $\vec{M}^{\sigma^{N E, \ell}}$ described above, and let $f(n)=\max \left(3 r q(n)(\ell(n)+m(n)), f^{\prime}(n)\right)$.

We now show that $\sigma_{1}^{*}, \sigma_{2}^{*}, \ldots$ is a subgame-perfect $(1 / q)$-equilibrium for every inverse polynomial discount factor $\delta \leq 1 / f$. We focus on deviations at histories of length $<\frac{n}{\delta(n)}$, since, by Lemma 3.5, the sum of payoffs received after that is negligible. Thus, there exists some $n_{0}$ such that, for all $n>n_{0}$, the payoff achieved after that history is less than $1 / q(n)$, which does not justify deviating.

We first show that no player has an incentive to deviate in subgames starting from phase 1 histories. By Lemma 4.4, it suffices to consider only a deviating strategy that after its first deviation deviates every time phase 1 is played; for every deviating strategy, either not deviating does at least as well or there is a deviating strategy of this form that does at least as well. Let $h_{1}$ be the history in which the deviation occurs and let $M$ be the deviating strategy. Notice that $\vec{M}^{\sigma^{N E, \ell}}$ can always act as intended at such histories; it can detect it is in such a history and can use the history to compute the next move (i.e., it does not need to maintain memory to figure out what to do next).

The player's payoff from $\left(M, \vec{M}_{-i}^{\sigma^{N E \ell}}\right)$ during one cycle of deviation and punishment can be at most $a$ at each round of phase 2 and, by Lemma 3.6, is negligible throughout phase 3. (We use $\epsilon_{n e g}$ to denote the negligible payoff to a deviator in phase 3.) Thus, the payoff of the deviating player from $\left(M, \vec{M}_{-i}^{\sigma^{N E, \ell}}\right)$ from the point of deviation onwards is at most

$$
\begin{gathered}
\left(\left(1-\delta(n)^{\left|h_{1}\right|}\right)\left(\delta(n)\left(m(n) a+\epsilon_{n e g}\right) \sum_{t=0}^{\left\lceil\frac{n f(n)-\left|h_{1}\right|}{m(n)+\ell(n)}\right\rceil}(1-\delta(n))^{(m(n)+\ell(n)) t}+\epsilon_{n e g}^{\prime}\right)\right. \\
\leq\left(\left(1-\delta(n)^{\left|h_{1}\right|}\right)\left(\delta(n)\left(m(n) a+\epsilon_{n e g}\right) \sum_{t=0}^{\infty}(1-\delta(n))^{(m(n)+\ell(n)) t}+\epsilon_{n e g}^{\prime}\right),\right.
\end{gathered}
$$

where $\epsilon_{n e g}^{\prime}$ is the expected payoff after round $n f(n)$. By Lemma 3.3. no matter where in the sequence the players are, the average discounted payoff at that point from playing honestly is at least $-1 / 2 q(n)$. Thus, the payoff from playing $\left(\vec{M}^{\sigma^{N E, \ell}}\right)$ from this point onwards is at least 
$\left.-(1-\delta(n))^{\left|h_{1}\right|}\right) 1 / 2 q(n)$. We can ignore any payoff before the deviation since it is the same whether or not the player deviates. and also divide both sides by $\left.(1-\delta(n))^{\left|h_{1}\right|}\right)$; thus, it suffices to prove that

$$
\delta(n)\left(m(n) a+\epsilon_{n e g}\right) \sum_{t=0}^{\infty}(1-\delta(n))^{(m(n)+\ell(n)) t}+\epsilon_{n e g}^{\prime} \leq \frac{1}{2 q(n)} .
$$

The term on the left side is bounded by $O\left(\frac{m(n) a+\epsilon_{n e g}}{n q(n)(m(n) a+1)}\right)$, and thus there exists $n_{1}$ such that, for all $n>n_{1}$, the term on the left side is smaller than $\frac{1}{2 q(n)}$ (In fact, for all constants $c$, there exists $n_{c}$ such that the left-hand side is at most $\frac{1}{c q(n)}$ for any $n>n_{c}$.)

We next show that no player wants to deviate in phase 2 or 3 histories. Notice that since these phases are carried out to completion even if the players deviate while in these phases (we do not punish them for that), and the honest strategy can easily detect whether it is in such a phase by looking at when the last deviation from phase 1 occurred. First consider a punishing player. By not following the strategy, he can gain at most $r$ for at most $\ell(n)+m(n)$ rounds over the payoff he gets with the original strategy (this is true even if his memory state is such that he just plays a fixed action, or even if another player deviates while the phase is played). Once the players start playing phase 1 again, our previous claim shows that no matter what the actual history is at that point, a strategy that does not follow the sequence does not gain much. It is easy to verify that, given the discount factor, a deviation can increase his discounted payoff by at most $\frac{1}{q(n)}$ in this case. (Notice that the previous claim works for any constant fraction of $1 / q(n)$, which is what we are using here since the deviation in the punishment phase gains $1 / c q(n)$ for some $c$.)

The punished player can deviate to a TM that correctly guessed the keys chosen (or the current TM's memory state might contain the actual keys and he defects to a TM that uses these keys) , in which case he would know exactly what the players are going to do while they are punishing him. Such a deviation exists once the keys have been played and are part of the history. Another deviation might be a result of the other TMs being in an inconsistent memory state, so that they play a fixed action, one which the deviating player might be able to take advantage of. However, these deviations work (or any other possible deviation) only for the current punishment phase. Once the players go back to playing phase 1, this player can not gain much by deviating from the sequence again. For if he deviates again, the other players will choose new random keys and a new random seed (and will have a consistent memory state); from our previous claims, this means that no strategy can gain much over a strategy that follows the sequence. Moreover, he can also gain at most $r$ for at most $\ell(n)+m(n)$ rounds which, as claimed before, means that his discounted payoff difference is less than $\frac{1}{q(n)}$ in this case.

This shows that, for $n$ sufficiently large, no player can gain more than $1 / q(n)$ from deviating at any history. Thus, this strategy is a subgame-perfect $1 / q$-equilibrium.

Using the same arguments as in Section 3.3 , we can also apply these ideas to efficiently find a computational subgame-perfect $\epsilon$-equilibrium in constant-degree graphical games.

Corollary 4.6. For all $a, b, d$, and all polynomials $q$, there is a polynomial $f$ and a polynomialtime algorithm $F$ such that, for all sequences $G_{1}, G_{2}, \ldots$ of games with $G^{j} \in G_{a, b, d, j, j}$ and for all inverse polynomials $\delta \leq 1 / f$, the sequence of outputs of $F$ given the sequence $G_{1}, G_{2}, \ldots$ of inputs is a subgame-perfect $\frac{1}{q}$-equilibrium for $G_{1}^{\infty}(\delta(1)), G_{2}^{\infty}(\delta(2)), \ldots$

\section{Acknowledgments}

Joseph Halpern and Lior Seeman are supported in part by NSF grants IIS-0911036 and CCF1214844, by AFOSR grant FA9550-08-1-0266, by ARO grant W911NF-14-1-0017, and by the Mul- 
tidisciplinary University Research Initiative (MURI) program administered by the AFOSR under grant FA9550-12-1-0040. Lior Seeman is partially supported by a grant from the Simons Foundation \#315783. Rafael Pass is supported in part by an Alfred P. Sloan Fellowship, a Microsoft Research Faculty Fellowship, NSF Awards CNS-1217821 and CCF-1214844, NSF CAREER Award CCF-0746990, AFOSR YIP Award FA9550-10-1-0093, and DARPA and AFRL under contract FA8750-11-2-0211. The views and conclusions contained in this document are those of the authors and should not be interpreted as representing the official policies, either expressed or implied, of the Defense Advanced Research Projects Agency or the US Government.

\section{References}

Andersen, G. and V. Conitzer (2013). Fast equilibrium computation for infinitely repeated games. In Twenty-Seventh AAAI Conference on Artificial Intelligence.

Aumann, R. and L. Shapley (1994). Long-term competitiona game-theoretic analysis. In N. Megiddo (Ed.), Essays in Game Theory, pp. 1-15. Springer New York.

Borgs, C., J. Chayes, N. Immorlica, A. Kalai, V. Mirrokni, and C. Papadimitriou (2010). The myth of the folk theorem. Games and Economic Behavior 70(1), 34-43.

Chen, X. and X. Deng (2006). Settling the complexity of two-player nash equilibrium. In Proc. 47th IEEE Symposium on Foundations of Computer Science, pp. 261-272.

Chen, X., X. Deng, and S. Teng (2006). Computing Nash equilibria: Approximation and smoothed complexity. In Proc. 47th IEEE Symposium on Foundations of Computer Science, pp. 603612.

Daskalakis, C., P. Goldberg, and C. Papadimitriou (2006). The complexity of computing a nash equilibrium. In Proc. 38th ACM Symposium on Theory of Computing, pp. 71-78.

Diffie, W. and M. Hellman (1976). New directions in cryptography. IEEE Transactions on Information Theory 22(6), 644-654.

Dodis, Y., S. Halevi, and T. Rabin (2000). A cryptographic solution to a game theoretic problem. In CRYPTO 2000: 20th International Cryptology Conference, pp. 112-130.

Fudenberg, D. and E. Maskin (1986). The folk theorem in repeated games with discounting or with incomplete information. Econometrica 54(3), 533-554.

Goldreich, O. (2001). Foundation of Cryptography, Volume I Basic Tools.

Goldreich, O., S. Goldwasser, and S. Micali (1986). How to construct random functions. Journal of the ACM 33(4), 792-807.

Goldwasser, S. and S. Micali (1984). Probabilistic encryption. Journal of Computer and System Sciences 28(2), 270-299.

Gossner, O. (1998). Repeated games played by cryptographically sophisticated players. Center for Operations Research \& Econometrics. Université catholique de Louvain.

Gossner, O. (2000). Sharing a long secret in a few public words. Technical report, THEMA (THéorie Economique, Modélisation et Applications), Université de Cergy-Pontoise.

Gradwohl, R., N. Livne, and A. Rosen (2013, January). Sequential rationality in cryptographic protocols. ACM Trans. Econ. Comput. 1(1), 2:1-2:38.

Halpern, J. Y. and R. Pass (2013). Sequential equilibrium in computational games. In Proc. 23rd International Joint Conference on Artificial Intelligence (IJCAI '13), pp. 171-176. 
Håstad, J., R. Impagliazzo, L. A. Levin, and M. Luby (1999). A pseudorandom generator from any one-way function. SIAM Journal on Computing 28(4), 1364-1396.

Hoeffding, W. (1963). Probability inequalities for sums of bounded random variables. Journal of the American Statistical Association 58(301), 13-30.

Jiang, A. X. and K. Leyton-Brown (2011). Polynomial-time computation of exact correlated equilibrium in compact games. In Proceedings of the 12th ACM conference on Electronic commerce, pp. 119-126. ACM.

Kearns, M., M. L. Littman, and S. P. Singh (2001). Graphical models for game theory. In Proc. Seventeenth Conference on Uncertainty in Artificial Intelligence (UAI 2001), pp. 253260.

Kol, G. and M. Naor (2008). Games for exchanging information. In Proc. 40th Annual ACM Symposium on Theory of Computing (STOC'08), pp. 423-432.

Kreps, D. M. and R. B. Wilson (1982). Sequential equilibria. Econometrica 50, 863-894.

Lehrer, E. (1991). Internal correlation in repeated games. International Journal of Game Theory 19(4), 431-456.

Littman, M. L. and P. Stone (2005). A polynomial-time nash equilibrium algorithm for repeated games. Decision Support Systems 39(1), 55-66.

Neyman, A. (1985). Bounded complexity justifies cooperation in the finitely repeated prisoners' dilemma. Economics Letters 19(3), 227-229.

Osborne, M. J. and A. Rubinstein (1994). A Course in Game Theory. Cambridge, Mass.: MIT Press.

Papadimitriou, C. H. and M. Yannakakis (1994). On complexity as bounded rationality. In Proc. 26th ACM Symposium on Theory of Computing, pp. 726-733.

Rivest, R. L., A. Shamir, and L. Adleman (1978). A method for obtaining digital signatures and public-key cryptosystems. Communications of the ACM 21(2), 120-126.

Rubinstein, A. (1979). Equilibrium in supergames with the overtaking criterion. Journal of Economic Theory 21(1), 1-9.

Rubinstein, A. (1986). Finite automata play the repeated prisoner's dilemma. Journal of Economic Theory 39(1), 83-96.

Selten, R. (1965). Spieltheoretische behandlung eines oligopolmodells mit nachfrageträgheit. Zeitschrift für Gesamte Staatswissenschaft 121, 301-324 and 667-689.

Urbano, A. and J. Vila (2004). Unmediated communication in repeated games with imperfect monitoring. Games and Economic Behavior 46(1), 143-173.

Urbano, A. and J. E. Vila (2002). Computational complexity and communication: Coordination in two-player games. Econometrica 70(5), 1893-1927.

\section{APPENDIX}

\section{A Multi-Instance PRFs}

In this section, we show that for any family of PRF, even polynomially many random members of it are indistinguishable from polynomially many truly random functions. 
Lemma A.1. For all polynomials $q$, if $\left\{f_{s}:\{0,1\}^{|s|} \rightarrow\{0,1\}^{|s|}\right\}_{s \in\{0,1\}^{*}}$ is a pseudorandom function ensemble, then the ensemble $F^{q}=\left\{F_{n}^{1}, \ldots, F_{n}^{q(n)}\right\}_{n \in \mathbb{N}}$ where, for all $i, F_{n}^{i}$ is uniformly distributed over the multiset $\left\{f_{s}\right\}_{s \in\{0,1\}^{n}}$, is computationally indistinguishable from $H^{q}=\left\{H_{n}^{1}, \ldots, H_{n}^{q(n)}\right\}_{n \in \mathbb{N}}$.

Proof. Assume for contradiction that the ensembles are distinguishable. This means there exist a polynomial $q$, a PPT $D$, and a polynomial $p$ such that for infinitely many $n$ 's

$$
\left|\operatorname{Pr}\left[D\left(1^{n},\left(H_{n}^{1}, \ldots, H_{n}^{q(n)}\right)\right)=1\right]-\operatorname{Pr}\left[D\left(1^{n},\left(F_{n}^{1}, \ldots, F_{n}^{q(n)}\right)\right)=1\right]\right|>\frac{1}{p(n)} .
$$

For each $n$, let $T_{n}^{i}=\left(1^{n},\left(H_{n}^{1}, \ldots, H_{n}^{i-1}, F_{n}^{i}, \ldots, F_{n}^{q(n)}\right)\right)$. We can now describe a PPT $D^{\prime}$ that distinguishes $\left\{F_{n}\right\}_{n \in \mathbb{N}}$ and $\left\{H_{n}\right\}_{n \in \mathbb{N}}$ for infinitely many $n$ 's. First notice that a PPT can easily simulate polynomially many oracle queries to both a truly random function and to a member of $F_{n}$. So $D^{\prime}$ on input $\left(1^{n}, X\right)$ randomly chooses $j \in\{1, \ldots, q(n)\}$ and calls $D$ with input $\left(1^{n},\left(I^{1}, \ldots, I^{j-1}, X, J^{j+1}, \ldots, J^{q(n)}\right)\right)$, where it simulates a query to $I_{k}$ as a query to a random member of $H_{n}$, and a query to $J_{k}$ as a query to a random member of $F_{n}$. (Notice that since $D$ is a PPT, it can make only polynomially many oracle queries to any of the functions, which can be easily simulated). Whenever $D$ makes an oracle query to $X, D^{\prime}$ makes an oracle query to $X$, and uses its answer as the answer to $D$. When $D$ terminates, $D^{\prime}$ outputs the same value as $D$.

Now notice that if $X$ is $H_{n}$, then the input to $D$ is $T_{n}^{j}$, while if $X$ is $F_{n}$, then the input to $D$ is $T_{n}^{j+1}$. Thus, $\operatorname{Pr}\left[D^{\prime}\left(1^{n}, H_{n}\right)=1\right]=\frac{1}{q(n)} \sum_{i=1}^{q(n)} \operatorname{Pr}\left[D\left(T_{n}^{i+1}\right)=1\right]$, and

$\operatorname{Pr}\left[D^{\prime}\left(1^{n}, F_{n}\right)=1\right]=\frac{1}{q(n)} \sum_{i=1}^{q(n)} \operatorname{Pr}\left[D\left(T_{n}^{i}\right)=1\right]$. It follows that

$$
\begin{aligned}
\left|\operatorname{Pr}\left[D^{\prime}\left(1^{n}, H_{n}\right)=1\right]-\operatorname{Pr}\left[D^{\prime}\left(1^{n}, F_{n}\right)=1\right]\right| & =\frac{1}{q(n)}\left|\sum_{i=1}^{q(n)} \operatorname{Pr}\left[D\left(T_{n}^{i+1}\right)=1\right]-\operatorname{Pr}\left[D\left(T_{n}^{i}\right)=1\right]\right| \\
& =\frac{1}{q(n)}\left|\operatorname{Pr}\left[D\left(T_{n}^{q(n)+1}\right)=1\right]-\operatorname{Pr}\left[D\left(T_{n}^{1}\right)=1\right]\right| \\
& >\frac{1}{q(n) p(n)},
\end{aligned}
$$

where the last inequality is due to the fact that $T_{n}^{q(n)+1}=\left(1^{n},\left(H_{n}^{1}, \ldots, H_{n}^{q(n)}\right)\right)$ and $T_{n}^{1}=\left(1^{n},\left(F_{n}^{1}, \ldots, F_{n}^{q(n)}\right)\right)$. But this means that for any such $n, D^{\prime}$ can distinguish $F=\left\{F_{n}\right\}_{n \in \mathbb{N}}$ and $H=\left\{H_{n}\right\}_{n \in \mathbb{N}}$ with non-negligible probability, and thus can do that for infinitely many $n$ 's. This is a contradiction to the assumption that $\left\{f_{s}:\{0,1\}^{|s|} \rightarrow\{0,1\}^{|s|}\right\}_{s \in\{0,1\}^{*}}$ is a pseudorandom function ensemble.

\section{B Multi-key Multi-Message Security}

In this section, we show that any secure public-key encryption scheme is also multi-key multimessage secure.

Lemma B.1. If (Gen, Enc, Dec) is a secure public key encryption scheme, then it is also multimessage multi-key secure.

Proof. Assume for contradiction that (Gen, Enc, Dec) is a secure public key encryption scheme that is not multi-message multi-key secure. Then there exist polynomials $f$ and $g$ and an adversary $A=\left(A_{1}, A_{2}\right)$ such that $\left\{\operatorname{IND}_{-M_{U L T}}^{\Pi}(A, k, f, g)\right\}_{k}$ and $\left\{\operatorname{IND}_{0} \operatorname{MULT}_{1}^{\Pi}(A, k, f, g)\right\}_{k}$ are distinguishable. That means there exist a PPT $D$ and a polynomial $p$ such that

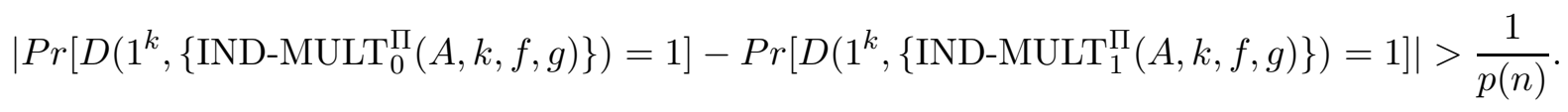


Let $T_{i, j}^{\pi}(A, k, f, g)$ be the following PPT algorithm:

$$
\begin{aligned}
T_{i, j}^{\pi}(A, k, f, g):= & \left(p k_{1}, s k_{1}\right) \leftarrow \operatorname{Gen}\left(1^{k}\right), \ldots\left(p k_{g(k)}, s k_{g(k)}\right) \leftarrow \operatorname{Gen}\left(1^{k}\right), \\
& \left(m_{0}^{1}, \ldots, m_{0}^{f(k)}, m_{1}^{1}, \ldots, m_{1}^{f(k)}, \tau\right) \leftarrow A_{1}\left(1^{k}, p k_{1}, \ldots, p k_{g(k)}\right) \\
& \mathcal{C} \leftarrow \operatorname{Enc}_{p k_{1}}\left(m_{0}^{1}\right), \ldots, \operatorname{Enc}_{p k_{g(k)}}\left(m_{0}^{1}\right), \\
& \ldots, \operatorname{Enc}_{p k_{1}}\left(m_{0}^{j}\right), \ldots, \operatorname{Enc}_{p k_{i-1}}\left(m_{0}^{j}\right), \operatorname{Enc}_{p k_{i}}\left(m_{1}^{j}\right), \ldots \operatorname{Enc}_{p k_{g(k)}}\left(m_{1}^{j}\right), \\
& \ldots \operatorname{Enc}_{p k_{1}}\left(m_{1}^{f(k)}\right), \ldots, \operatorname{Enc}_{p k_{g(k)}}\left(m_{1}^{f(k)}\right) \\
& o \leftarrow A_{2}(\mathcal{C}, \tau) \\
& \text { Output } o .
\end{aligned}
$$

We now define an adversary $A^{\prime}=\left(A_{1}^{\prime}, A_{2}^{\prime}\right)$, and show that $\left\{\operatorname{IND}_{0}^{\Pi}\left(A^{\prime}, k, f, g\right)\right\}_{k}$ and $\left\{\operatorname{IND}_{1}^{\Pi}\left(A^{\prime}, k, f, g\right)\right\}_{k}$ are not computationally indistinguishable. $A_{1}^{\prime}$ on input $\left(1^{k}, p k\right)$ first chooses $i \in\{1, \ldots, g(k)\}$ uniformly at random. It then generates $g(k)-1$ random key pairs $\left(p k_{1}, s k_{1}\right), \ldots,\left(p k_{i-1}, s k_{i-1}\right),\left(p k_{i+1}, s k_{i+1}\right), \ldots,\left(p k_{g(k)}, s k_{g(k)}\right)$. It then calls $A_{1}$ with input $\left(1^{k}, p k_{1}, \ldots, p k_{i-1}, p k, p k_{i+1}, \ldots, p k_{g(k)}\right)$. After getting $A_{1}$ 's output $M=\left(m_{0}^{1}, \ldots, m_{0}^{f(k)}, m_{1}^{1}, \ldots, m_{1}^{f(k)}, \tau\right), A_{1}^{\prime}$ chooses $j \in\{1, \ldots, f(n)\}$ uniformly at random, and returns as its output $\left(m_{0}^{j}, m_{1}^{j},\left(i, j, p k, p k_{1}, s k_{1}, \ldots, p k_{g(k)}, s k_{g(k)}, M\right)\right)$.

$A_{2}^{\prime}$ on input $\left(\mathcal{C},\left(i, j, p k, p k_{1}, s k_{1}, \ldots, p k_{g(k)}, s k_{g(k)}, M\right)\right)$ constructs input $\mathcal{C}^{\prime}$ for $A_{2}$ by first appending the encryptions of messages $m_{0}^{1} \ldots, m_{0}^{j-1}$ with all the keys, then appending the encryption of $m_{0}^{j}$ with keys $p k_{1}, \ldots, p k_{i}$ and then appends $\mathcal{C}$. It then appends the encryption of $m_{1}^{j}$ with keys $p k_{i+2}, \ldots, p k_{g(k)}$ and also the encryption of the messages $m_{1}^{j+1}, \ldots, m_{1}^{f(k)}$ with each of the keys. It then outputs $A_{2}\left(\mathcal{C}^{\prime}, \tau\right)$. If $\mathcal{C}$ is the encryption of $m_{j}^{0}$ with key $p k$, then this algorithm is identical to $T_{i+1, j}^{\pi}(A, k, f, g)$ (if $i=g(k)$ then by $T_{i+1, j}^{\pi}$ we mean $T_{1, j+1}^{\pi}$; we use similar conventions elsewhere), while if it is the encryption of $m_{j}^{1}$ with key $p k$, then the algorithm is identical to $T_{i, j}^{\pi}(A, k, f, g)$.

We claim that $D$ can distinguish $\left\{\operatorname{IND}_{0}^{\Pi}\left(A^{\prime}, k, f, g\right)\right\}_{k}$ and $\left\{\operatorname{IND}_{1}^{\Pi}\left(A^{\prime}, k, f, g\right)\right\}_{k}$. Note that

$$
\operatorname{Pr}\left[D\left(1^{k},\left\{\operatorname{IND}_{0}^{\Pi}\left(A^{\prime}, k, f, g\right)\right\}\right)=1\right]=\frac{1}{g(k) f(k)} \sum_{j=1}^{f(k)} \sum_{i=1}^{g(k)} \operatorname{Pr}\left[D\left(1^{k}, T_{i+1, j}^{\pi}(A, k, f, g)\right)=1\right]
$$

and

$$
\operatorname{Pr}\left[D\left(1^{k},\left\{\operatorname{IND}_{1}^{\Pi}\left(A^{\prime}, k, f, g\right)\right\}\right)=1\right]=\frac{1}{g(k) f(k)} \sum_{j=1}^{f(k)} \sum_{i=1}^{g(k)} \operatorname{Pr}\left[D\left(1^{k}, T_{i, j}^{\pi}(A, k, f, g)\right)=1\right] .
$$

Thus,

$$
\begin{aligned}
& \left|\operatorname{Pr}\left[D\left(1^{k},\left\{\operatorname{IND}_{0}^{\Pi}\left(A^{\prime}, k, f, g\right)\right\}\right)=1\right]-\operatorname{Pr}\left[D\left(1^{k},\left\{\operatorname{IND}_{1}^{\Pi}\left(A^{\prime}, k, f, g\right)\right\}\right)=1\right]\right| \\
= & \frac{1}{g(k) f(k)} \mid \sum_{j=1}^{f(k)} \sum_{i=1}^{g(k)}\left(\operatorname{Pr}\left[D\left(1^{k}, T_{i+1, j}^{\pi}(A, k, f, g)\right)=1\right]-\operatorname{Pr}\left[D\left(1^{k}, T_{i, j}^{\pi}(A, k, f, g)\right)=1\right]\right) \\
= & \frac{1}{g(k) f(k)}\left|\operatorname{Pr}\left[D\left(1^{k},\left\{\operatorname{IND}_{-} \operatorname{MULT}_{0}^{\Pi}(A, k, f, g)\right\}\right)=1\right]-\operatorname{Pr}\left[D\left(1^{k},\left\{\operatorname{IND}_{-} \operatorname{MULT}_{1}^{\Pi}(A, k, f, g)\right\}\right)=1\right]\right| \\
> & \frac{1}{g(k) f(k) p(k)},
\end{aligned}
$$

where the next-to-last line follows because $T_{1,1}^{\pi}(A, k, f, g)=\operatorname{IND}_{-M_{U L T}}^{\Pi}(A, k, f, g)$ and

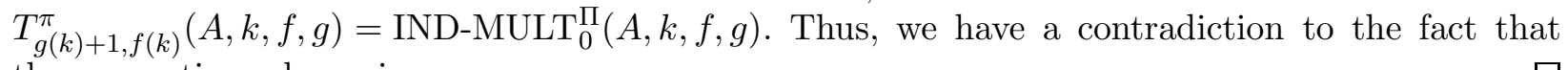
the encryption scheme is secure. 